\title{
Modeling Binocular and Motion Transparency Processing by Local Center-Surround Interactions
}

\author{
Florian Raudies \\ Department of Cognitive and Neural Systems, Boston University, Boston, MA, 02215, USA \\ Heiko Neumann \\ Institute of Neural Information Processing, University of Ulm, 89069 Ulm, Germany
}

\begin{abstract}
Binocular transparency is perceived if two surfaces are seen in the same spatial location, but in different depths. Similarly, motion transparency occurs if two surfaces move differently over the same spatial location. Most models of motion or stereo processing incorporate uniqueness assumptions to resolve ambiguities of disparity or motion estimates and, thus, can not represent multiple features at the same spatial location. Unlike these previous models, we suggest a model with local center-surround interaction that operates upon analogs of cell populations in velocity or disparity domain of the ventral second visual area (V2) and dorsal medial middle temporal area (MT) in primates, respectively. These modeled cell populations can encode motion and binocular transparency. Model simulations demonstrate the successful processing of scenes with opaque and transparent materials, not previously reported. Our results suggest that motion and stereo processing both employ local center-surround interactions to resolve noisy and ambiguous disparity or motion input from initial correlations.
\end{abstract}

\section{INTRODUCTION}

In real world situations transparency and semi-transparency often occur as likely as opaque surfaces in different depths or moving differently. For instance, reflections in the window of a driving vehicle overlay with the background outside the car. Driving with a dirty windshield, where the dust on the windshield is moving independently from the outside world creates two layers of independent motion in the same region of the visual field. Also, semi-transparency occurs, for instance, when viewing crowds of people moving in stripes of opposite directions from an elevated position. Here, by spatial and temporal integration of motion signals in the visual system, two motions are perceived at the same spatial locations of the visual field.

Although transparency and semi-transparency configurations occur in real-world situations standard models of stereo and motion processing typically do not support the processing of transparent surfaces. Instead, these models assume the existence of a single computational solution generating a single depth representation or motion quantity for each spatial location. Thus, the majority of neural models do not successfully explain the processing of transparent and opaque surfaces. This motivated the development of a new generalized model for binocular and motion transparency processing using essentially the same computational mechanisms operating upon different feature domains. These proposed mechanisms and their representations are inspired by recent reports of neural populations in different visual areas and their selectivity to orientation, spatial frequency, disparity, and visual motion. These neural populations can encode multiple stimulus disparities or motions (Treue et al., 2000). Our objectives for the newly proposed model are severalfold. First, we suggest that the perception of transparent (as well as opaque) motion and stereo stimuli can be explained by the same mechanism of local center-surround interaction. Second, the suggested mechanisms of our model architecture are in 
accordance with the general physiology on disparity and motion processing and show coinciding tuning properties of the model's population with recorded data. Finally, the model is probed with realistic image sequences and results demonstrate its successful application for computational vision.

\section{BACKGROUND}

\section{Perception and Neurophysiology of Binocular and Motion Transparency}

The conditions under which binocular and motion transparency occur have been extensively studied in psychophysics and neurophysiology. For instance, the number of planes in random dot stereograms (RDS) at different depths that could be perceived in parallel is reported to vary between three and six (Tsirlin et al., 2008; Reeves et al., 2010). A closer look into the arrangement of planes at different distances (measured in visual degrees) shows that for values below approximately three min arc the distance is underestimated, above three min arc the distance is overestimated for a wide range of angular separations (Stevenson et al., 1991). This mis-estimation is known as the effect of attraction and repulsion in perception.

The processing of motion transparency shares several properties with that of binocular transparency. For instance, the angular difference between two motion components in a random dot kinematogram (RDK) is perceived as smaller when directions differ less than $25^{\circ}$ (motion direction attraction). When directions differ by more than $40^{\circ}$ the difference is perceived as larger (motion direction repulsion). Veridical motion is perceived when motion directions differ by more than $135^{\circ}$ (Edwards \& Nishida, 1999; Braddick et al., 2002). Two components of line gratings with different speed can be perceived as transparently overlaid (Meso \& Zanker, 2009). Two to three motions of different direction in RDKs can be perceived as transparently overlaid (Mulligan, 1992; Felisberti \& Zanker, 2005; Greenwood \& Edwards, 2009). Neural responses for RDKs with two motion components of different angle have a single peak for small angular differences and multiple peaks for angles above $\sim 60^{\circ}$ (Treue et al., 2000). Cells in the primary visual cortex (area V1) can represent more than one motion direction, e.g. by using different subpopulations. Cells in middle temporal area (MT) strongly respond to the preferred motion direction and weakly to the anti-preferred motion direction, although both directions are equally present in the stimulus RDK (Snowden et al. 1991). In sum, these findings provide hints about the underlying neural representations and mechanism employed in motion and stereo perception. Furthermore, these findings suggest that the occurrence of binocular and motion transparency is based on similar neural mechanisms to achieve effects similar in both domains, like the effect of attraction and repulsion.

\section{Methods for Binocular and Motion (Transparency) Processing}

Methods of binocular or motion transparency processing have to solve three intertwined problems: the spatial and temporal integration, the segregation, and the clustering of different stimulus components into, e.g., separate depth planes or motions. Visual motion signals have to be spatio-temporally integrated. Binocular stereo signals are spatially integrated and can profit from a temporal integration as well. But while integrating components, those originating from different objects in depth or with different motions have to remain separate. For instance, two planes, one transparent and leftward moving at close distance, another opaque and rightward moving at far distance, have to be kept segregated while integrating their belonging motion and depth signals. Otherwise the overall motion would be zero, assuming the same motion speed for both planes, and the extracted depth would be the mean of near and far, assuming a $50 \%$ transparency for the near plane. In contrast to the segregation, the clustering refers to the estimation of the number of components, e.g. that two motions exist in the above example. In general, the estimate of surface properties from noisy and ambiguous input profits from a uniqueness constraint implementing the assumption about a single component in one spatial location. However, transparency requires the detection and representation of multiple components which contradicts the uniqueness assumption.

In the binocular stereo domain the correspondence problem has to be solved, that is the identification and matching of the same point location between left and right eye's image. An early model 
for matching is the disparity energy model, which was inspired by the complex cell responses in cat's visual cortex (Ohzawa et al., 1990). Disparity energy is detected by four simple cells, two for each dominant eye input, whereas the two cells receiving input from one eye have a $90^{\circ}$ phase difference. Responses of single cells are modeled by Gabor filtering of the input image of a stereo pair. Responses of the same phase are summed and squared for both phase signals. Finally, the two squared signals for the different phases are summed and the square root is taken. These computational steps define the neural model circuit of a disparity detector. Note that this circuit is the same that has been used for the motion energy detector (Adelson \& Bergen, 1985). The disparity detector used together with a phase-mechanism, varying the phase for the left and right eye's image, allows the computation of likelihood maps for different disparity values ${ }^{i}$ (Qian, 1994). Due to the constraints set by the sampling theorem (Nyquist, 2002) phase-shift based disparity detectors are always limited to $\pm \lambda / 2$ with $\lambda$ denoting the wavelength of the Gabor filter. Thus, the detection of large disparities requires Gabor filters with large wavelengths and large standard deviation. Subsequently, their detection works only on a low spatial resolution. To overcome this limitation, a phase-shift mechanism can be combined with a position-shift mechanism (Chen \& Qian, 2004). In principle, the disparity detector approach can detect multiple pointcorrespondences for a single spatial location and, thus, potentially support binocular transparency.

Besides the methods for initial correspondence finding, different schemes of interaction between disparities and neighboring spatial locations have been proposed. A cooperative competitive scheme forces uniqueness for all possible point matches, the disparities at one location, and assumes continuity to support similar disparity values at spatially adjacent locations (Marr \& Poggio, 1976). Both rules, uniqueness and continuity, are embedded into an iterative scheme of local interaction between nodes representing the likelihood of a match of neighboring locations and different disparities at one location. Stability between these two rules is achieved after view iterations. The uniqueness constraint is criticized for not supporting multiple matches at a single location, namely binocular transparency (Weinshall, 1989). Another scheme of interaction that relaxes the uniqueness constraint was suggested by Prazdny (1985). This interaction uses a Gaussian function with the disparity distance divided by the distance of the referring pixel locations as argument (see Equation 1 in Prazdny, 1985). For a fixed disparity difference this leads to increased support if the reference locations are farther apart. In general this relaxed interaction scheme allows the representation of multiple activations at the same location or binocular transparency. A network trained with a modified back-prop learning rule based on sparse compatibility maps (the correspondences of locations with an intensity value of one in the RDS) was able to infer the uniqueness and continuity constraints (Qian \& Sejnowski, 1989). The trained network showed reasonable results also for sparse transparency stimuli. Furthermore, Pollard and Frisby demonstrated that RDSs with binocular transparency can be successfully processed by using a competitive scheme that includes a uniqueness constraint (Pollard \& Frisby, 1990). How could uniqueness rule out transparency and still give reasonable results for RDSs including transparency? For Pollard and Frisby's simulations only the pooled response over all image rows was reported, essentially leaving open if multiple correspondences for single locations were existent. In order to account for the perceptual performance of humans it remains which spatial resolution or pooling size is required to distinguish between semi-transparency and transparency in the stereo or motion domain.

Another form of interaction is defined by a coarse-to-fine scheme which includes the detection of disparity shifts or visual motions at multiple spatial resolutions. One possible incorporation of a coarse-tofine scheme into a phase-shift and position-shift based model is the estimation of phase-shifts at each scale, whereas the estimate from the coarsest scale is propagated to finer scales by using the position-shift mechanism (Chen \& Qian, 2004). The disparity energy model with a phase-shift mechanism for multiple spatial resolutions along with a template matching for read-out can account for binocular transparency (Tsai \& Victor, 2003; Watanabe, 2008).

Similar to the approaches in the stereo domain, motion models use different detectors for the initial estimation of motion signals and similar interaction schemes for the spatio-temporal integration. Initial motion is detected by the Hassenstein-Reichardt detectors (Hassenstein and Reichardt, 1956), motion energy filters (Adelson and Bergen, 1985), also applied in the frequency domain (Heeger, 1987), 
or multi-channel gradients (Johnston et al., 1999). Furthermore, motion transparency processing has been analyzed for the Hassenstein-Reichardt detector (Zanker, 2005), motion energy filtering (Qian et al., 1994; Simoncelli and Heeger, 1998, Watanabe and Kikuchi, 2005), and multi-channel gradients (Durant et al., 2006). A simple interaction scheme is the pooling of initial motion signals over the entire visual space (Zanker, 2005; Durant et al., 2006), while advanced schemes use competitive mechanisms between opponent motion-directions (Qian et al., 1994b) or cross-talk between segregated responses attached to stimulus parts / motions (Watanabe and Kikuchi, 2005). This shows the remarkable similarity between models of binocular stereo and motion processing, using comparable detectors and schemes of interaction for spatio-temporal integration.

Computer vision algorithms segregate motions using parametric motion models that are simultaneously fitted to the data in space. These models are spatially and piecewise fitted to deformations and motions of the incoming image sequence (Darrell and Pentland, 1991; Langley et al., 1992; Darrell and Simoncelli, 1993; Black and Anandan, 1993, 1996; Ju et al., 1996), multiple motion models are fitted to the image sequence by using variational approaches (Ramirez-Manzanares et al., 2006), or a set of spatio-temporal filters is applied to extract two motions for each pixel (Liu et al., 1995). Integration in the visual field is achieved by incorporating spatio-temporal smoothness constraints which assume that no strong changes in motions occur at nearby spatial locations through time (Horn and Schunk, 1981). To cluster motions $n$ th-order derivatives are combined (Langley et al., 1992) by utilizing a generalized structure tensor defined as the covariance of spatio-temporal derivatives of the same order. The rank of this tensor matrix determines the number of motions (Mota et al., 2004). To sum up, yet there does not exist a coherent model that deals with motion and stereoscopic input data from (semi-) transparent as well as opaque surface configurations that can be processed in a coherent architecture of visual processing. Here, we propose common mechanisms for stereo and motion processing defined on the basis of findings about the structure and function of the primate visual system. In the next paragraphs we develop these common mechanisms for the detection and integration of binocular stereo and motion signals, starting with an overview, then presenting correlation detectors for disparity and motion signals, proposing a three-step-processing cascade of layered neural processing, that is replicated in all three model areas, and the incorporation of local center-surround interactions enabling and stabilizing multiple activations for transparency stimuli.

\section{A CORTICAL MODEL FOR BINOCULAR AND MOTION TRANSPARENCY PROCESSING}

Our model resembles the cortical mechanisms of V1, second visual area (V2), and MT for the processing of stereo and motion stimuli, see Figure 1a. Model area V1 includes correlation detectors for spatially offset locations as well as temporally delayed signals. Initial correlation results are linked into a threestep-processing cascade that is replicated in model areas V2 and MT. Model areas V1 and V2 define the stereo signal processing pathway, containing a population of model neurons sensitive to disparity. Model areas V1 and MT define the processing pathway for visual image motion. Although there is an overlap between both pathways in area V1, they are kept mutually exclusive and, thus, do not interact. Note that area MT neurons show sensitivity to stereo signals as well (Born \& Bradley, 2005); however, we used the traditional view of V2 being pathway for stereo vision (e.g., Hubel \& Wiesel, 1970; Hubel \& Livingstone, 1987). Both modeled pathways incorporate bidirectional connections between areas V1 and V2 for stereo signaling and V1 and MT for motion signaling. Feedback (FB) signals as part of the bidirectional connectivity modulate ascending, driving signals. In all model areas disparity and motion signals are represented in populations of neurons, selective to disparity and motion, respectively. These populations can represent multiple activations at a single spatial location through the formation of multiple local peaks in the activity distribution. Thus, these codes provide a solution to the problem of clustering; the estimation of the number of motions or disparities at a single location. The next paragraphs contain a formal definition and explanation for the initial correlation mechanisms to derive estimates, and the threestage processing cascades in model areas V1, V2, and MT. 
Figure 1. Model visual areas and mechanism supporting transparency processing. (a) The suggested model resembles functions of primate visual areas V1,V2, and MT. Local correspondences for stereo and motion are computed in area V1 resulting in disparity and motion direction selectivity analog to neurons in V1. Stereo signals are integrated in area V2 and motion signals in area MT. (b) Binocular transparency and (c) motion transparency are supported by local center-surround interactions for disparity and motion speeds / directions, respectively. Local competition is depicted by the light gray ellipse to denote excitatory connectivity and the dark gray ellipse to denote inhibitory connectivity with reference to the left eye's disparity or motion at the center of the ellipses.

\section{Spatial and spatio-temporal correlation for the detection of initial stereo and motion}

signals. Assume that the image intensity signal for left and right image is given by the function $f^{L / R}(x, y)$ for the 2D image coordinates $(x, y)$. The superscripts $L$ and $R$ denote the left eye's image and right eye's image, respectively. The stereo correlation signal is computed in five steps. First, the direct current (DC) component of left and right eye's input image is removed, defining

$$
\bar{f}^{L / R}(x, y)=f^{L / R}(x, y)-\frac{1}{\left|I_{x, y}\right|} \sum_{x, y \in I_{x, y}} f^{L / R}(x, y),
$$

where the second term on the right hand side denotes the mean value computed over all spatial locations $x$ and $y$ of the image domain $\mathrm{I}_{\mathrm{x}, \mathrm{y}}$. Second, orientation specific response maps

$$
r^{L / R}\left(x, y, \psi_{k}\right)=\bar{f}^{L / R}(x, y) * f^{\text {Gauss }}\left(x, y ; \psi_{k}\right)
$$

are constructed by convolving the DC free input signal with the orientation specific Gaussian filter $f^{\text {Gauss }}\left(x, y ; \psi_{k}\right)=\sin \left(\psi_{k}\right) \cdot \partial_{x} f^{\text {Gauss }}(x, y)+\cos \left(\psi_{k}\right) \cdot \partial_{y} f^{\text {Gauss }}(x, y)$, using the steerable filter theorem (Freeman \& Adelson, 1991). The symbol * in Eqn.(2) denotes the convolution operator. Partial derivatives are denoted by $\partial_{x}$ and $\partial_{y}$ for the $x$ and $y$ dimension, respectively. These derivatives are numerically approximated by applying Sobel operators (Danielson \& Segera, 1990). This oriented Gaussian filter approximates a Gabor filter (Daugman, 1985) with unit wavelength. Note that the selectivity of area V1 neurons has been characterized by using Gabor filters as a parameterized model (Ringach, 2002). All parameters of our model are reported in Table 1. Third, the normalization over orientations

$$
\bar{r}^{L / R}\left(x, y, \Psi_{k}\right)=r^{L / R}\left(x, y, \Psi_{k}\right) /\left(\eta_{N}+\frac{1}{N_{\text {ori }}} \sum_{k=1}^{N_{\text {ori }}} r^{L / R}\left(x, y, \Psi_{k}\right)\right)
$$

generates a signal that is independent of local brightness changes, which has been previously used in motion detectors (Heeger, 1987; Bayerl \& Neumann, 2004). Fourth, these orientation maps are compared

$$
c^{L}(x, y ; \Delta x)=\frac{1}{N_{\text {ori }}} \cdot \sum_{k=1}^{N_{\text {ori }}} \exp \left(-\left[\bar{r}^{L}\left(x, y ; \Psi_{k}\right)-r^{R}\left(x-\Delta x, y ; \Psi_{k}\right)\right]^{2} /\left(2 \cdot \sigma_{c}{ }^{2}\right)\right)
$$

for different horizontal disparities $\Delta x$ between the left eye's (reference) and right eye's image by using a Gaussian function to rate pixel-similarity. Note that this similarity measure is inverse to the typically defined matching cost functions, such as squared difference, absolute difference, or wavelet phase (Scharstein \& Szeliski, 2002). Individual pixel-similarities of each orientation are summed to improve the signal-to-noise ratio. For further improvement, the left eye's image is compared with the right eye's one in the same way, where the right eye's image serves as reference. Then pixel-similarity for the right eye's image are registered with those of the left eye's one by warping the pixel-similarity to the left according to the estimated horizontal disparity. Fifth, the left eye's and the registered right eye's pixel similarity are summed

$$
\bar{c}^{L}(x, y ; \Delta x)=1 / 2 \cdot\left(c^{L}(x, y, \Delta x)+c^{R}(x-\Delta x, y, \Delta x)\right)
$$


leading to the final correlation result with reference to the left image. Due to the missing pixelcorrespondences at the left boundary, where no matching parts in the right image exist, these values in the non-match region are homogenously set to one. Later in the interaction scheme these values can be modulated by nearby spatial correlations.

Initial motion signals are detected by using a correlation mechanism of local image-phases. Motion sequences are defined as varying brightness changes by the function $f(x, y, t)$ with the spatial coordinates $x$ and $y$, and the temporal coordinate $t$. Note that this image sequence refers to a generic image stream not directly bound to the left or right eye. However, in the simulations visual motions for stereo image sequences are computed with respect to the left eye's image. Input images of the sequence are convolved with Gabor filters of different spatial resolution according to the spatial shift values or visual motion speeds tested. These Gabor filters resemble the variety of neural selectivity of area V1 neurons better than the above oriented Gaussian filters (Ringach, 2002). The convolution of images from the motion sequence with Gabor filters is performed in the Fourier domain. Therefore, individual frames of the image sequence $f(x, y, t)$ are Fourier transformed into $\hat{f}\left(\omega_{x}, \omega_{y}, t\right)$ which is indicated by the hatsymbol and the change of arguments to angular frequencies $\omega_{\mathrm{x}}$ and $\omega_{\mathrm{y}}$, corresponding to the spatial dimensions. The temporal dimension is not Fourier transformed. The Gabor filters for different spatial resolutions are defined in the Fourier domain as

$$
\hat{f}^{\text {Gabor }}\left(\omega_{x}, \omega_{y} ; \psi_{k}, \omega_{l}, \sigma_{l}^{\psi}, \sigma_{l}^{r}\right)=\frac{1}{2 \cdot \pi \cdot \sigma_{l}^{\psi} \cdot \sigma_{l}^{r}} \cdot \exp \left(-\frac{1}{2}\left[\left(\frac{\omega_{r}+\omega_{l}}{\sigma_{l}^{r}}\right)^{2}+\left(\frac{\omega_{\psi}}{\sigma_{l}^{\psi}}\right)^{2}\right]\right)
$$

by using Gaussians as window functions centered at the angular frequencies $\omega_{r}+\omega_{l}$ and $\omega_{\psi}$ in angular coordinates. These angular coordinates are defined by $\omega_{r}=\cos \left(\psi_{k}\right) \cdot \omega_{x}-\sin \left(\psi_{k}\right) \cdot \omega_{y}$ and $\omega_{\psi}=\sin \left(\psi_{k}\right) \cdot \omega_{x}+\cos \left(\psi_{k}\right) \cdot \omega_{y}$, rotating the x-axis and y-axis of the Fourier space into the orientation $\psi_{k}$. The Gabor filters for different spatial resolutions are denoted by index $l$ and those for different orientations by index $k$. The angular frequency of the Gabor filters is defined by $\omega_{l}$. A filtering response for a Gabor filter is given by

$$
r\left(x, y, t ; \psi_{k}, \omega_{l}, \sigma_{l}^{\psi}, \sigma_{l}^{r}\right) \quad \circ-F\{f(x, y)\}-\bullet \hat{f}\left(\omega_{x}, \omega_{y}, t\right) \cdot \hat{f}^{\text {Gabor }}\left(\omega_{x}, \omega_{y} ; \psi_{k}, \omega_{l}, \sigma_{l}^{\psi}, \sigma_{l}^{r}\right)
$$

which uses the inverse Fourier transform $F$ for the spatial dimension and the result are complex numbers. These complex numbers are used to define a local signal amplitude and local signal phase $\Phi_{k, l, t}=\arg \left\{r\left(x, y ; \psi_{k}, \omega_{l}, \sigma_{l}^{\psi}, \sigma_{l}^{r}\right)\right\}$. In our correlation detector only the local image phase is further used, because it is largely independent of local brightness variations and, thus, provides a signature of a local image region mainly independent of the overall brightness level. Therefore, a local normalization like for the disparity detector in Equation (3) is not required. Neurons in area V1 exist that encode local image phase (Mechler et al., 2002). Motion signals are calculated by using the extended HassensteinReichardt detector (Hassenstein \& Reichardt, 1956) estimating spatio-temporal correlations of local image phase. A local image phase

$$
\Phi_{k, l, t}^{\Delta m, l}=\arg \left\{r\left(x-\Delta x_{l, m}, y-\Delta y_{l, m} ; \psi_{k}, \omega_{l}, \sigma_{l}^{\psi}, \sigma_{l}^{r}\right)\right\}
$$

is spatially shifted by the horizontal and vertical offsets $\Delta x_{l, m}=s_{l} \cdot \cos \left(\phi_{m}\right)$ and $\Delta y_{l, m}=s_{l} \cdot \sin \left(\phi_{m}\right)$ and compared against the non-shifted but temporally delayed phase. For the forwards correlation from time $t_{0}$ to $t_{1}$ the detection is given by

$$
c_{l, m}^{t_{0} \rightarrow t_{1}}=\frac{1}{N_{\text {ori }}} \sum_{k=1}^{N_{\text {ori }}}\left[\cos \left(\Phi_{k, l, t_{1}}^{\Delta m, l}-\Phi_{k, l, t_{0}}\right)\right]^{+},
$$

with $[\cdot]^{+}=\max (\cdot, 0)$ denoting a half-wave rectification operation and the correlation results for all orientations is summed ${ }^{\mathrm{ii}}$. The rectified cosine-function defines the tuning between phase differences. The 
backwards correlation $c_{l, m}^{L, t_{1} \rightarrow t_{0}}$ is analogously defined. Forwards and backwards correlation are combined by subtracting the backwards correlation from the forwards correlation,

$$
c\left(x, y, \phi_{m}, s_{l}\right)=\left[c_{l, m}^{t_{1} \rightarrow t_{0}}-c_{l, m}^{t_{0} \rightarrow t_{1}}\right]^{+},
$$

because they appear in common only for flicker motion which should not be detected.

Different speed and disparity ranges. The proposed disparity and motion correlation detectors use different filters due to the difference in the range of detected disparities and motion speeds. Typical benchmarks use disparity values between zero and 60 pixels; however, motion speeds rarely are above six pixels per frame. As we use a logarithmic scale to sample motion speeds (see Table 1), large disparities could be only sparsely sampled by utilizing the same filtering mechanisms in the disparity domain. Although selectivity for disparity values in areas V1 and V2 might be logarithmically sampled in cortex, benchmark sequences require a dense sampling of disparities over a wide range. Thus, instead of applying a Gabor filter bank with various spatial resolutions for the detection of horizontal disparities based on local image phase as we did for the motion detector disparity is detected by using a pixel-wise similarity measure, defined in Equation 4.

Neurons in area V1 show a phase and shift selectivity (Prince et al., 2002) for different disparities capturing a spatial shift either by a shift of the entire Gabor filter or only by shifting its phase while the Gaussian support of the Gabor filter remains at the same location. Our model only includes shift selective neurons. Furthermore, only absolute disparity signals are encoded in our model area V2, excluding the encoding of relative disparity as found in V2 neurons (Thomas et al., 2002). Absolute disparity signals are encoded in model V1 as well as in V1 of macaque monkeys (Cumming \& Parker, 1999).

Visual motion speeds and motion directions are encoded in areas V1 and MT of macaque monkey (Priebe et al., 2006; Cheng et al., 1994; Orban et al., 1986; Rodman \& Albright, 1987). Motion speed and direction selectivity in our model are generated by spatio-temporally correlating local image phases extracted by Gabor filters of different orientation and spatial resolution. The relationship between the wavelength of the Gabor filter (spatial frequency) and the different spatial shifts to be tested are crucial mechanisms for our detector model. Spatial shifts have to be below half of the wavelength in accordance to the sampling theorem (Nyquist, 2002).

Due to the two frame correlation employed for simplicity, the motion detector does not detect different temporal frequencies. Instead spatio-temporal selectivity is generated by detecting different spatial frequencies for one temporal frequency. The corresponding image velocity is computed as $v=f(t) / f(x)$ where $f(t)$ denotes the temporal frequency and $f(x)$ denotes the spatial frequency. Measured cells and model cells both have a Gaussian-shaped tuning curve in the velocity domain (motion directions and speeds), where speeds are encoded on a logarithmic scale in area MT (Maunsell and Essen, 1983, compare with their Figure 6-A); Nover et al., 2005).

Three-step processing cascade for model area V1. The three-stage processing cascade is motivated by the layered cortical processing within visual cortex. The first processing step includes a spatial integration and nonlinear signal enhancement, which takes place through signal processing in synapses and dendrites by the integration of incoming signals and is defined by the ordinary differential equation (ODE) ${ }^{\text {iii }}$

$$
\dot{x}_{V 1}^{(1)}=-x_{V 1}^{(1)}+\left(x_{V 1}^{F F}\right)^{\alpha} * \Lambda_{d i s p / m o t} .
$$

The signals $x_{V 1}^{(1)}$ and $x_{V 1}^{F F}$ model the membrane potential, or more generally the activity, of a cell or firing rate (Stafstrom et al., 1984). This activation encodes the likelihood for a disparity or motion in the stimulus input. The signals $x_{V 1}^{F F}$ from the initial correlation, $x_{V 1}^{F F}=\bar{c}^{L}$ from Equation 5 for the stereo signaling pathway or $x_{V 1}^{F F}=c$ from Equation 10 for the motion signaling pathway, are first transformed by a non-linear function to model the nonlinearities of dendrites and synapses in signal processing. Here, 
we employ an exponential nonlinearity with $\alpha>1$ as exponent. The signal is then convolved with the Gaussian filter $\Lambda_{\text {disp /mot }}$, where convolution takes place over either the disparity or motion domain. Note that these convolutions define an interaction between point processes of model cells.

In the second processing step FB signals modulate driving forward signals, where FB originates from visual areas higher in the hierarchy than the considered target area. By integrating over larger spatial regions of visual space in higher visual areas, FB can provide disambiguated signals and broader context to shape the neural activity of early visual areas through the modulation of cell activities whose feature selectivity matches the representation built at the higher level (e.g. area) in the cortical hierarchy of visual motion processing (Bullier et al., 2001). A modeling study details the properties of such a modulation, defined as the linking principle (Eckhorn et al., 1990). Linking suggests that the driving signal can be enhanced by FB, but FB alone can not generate activity, it always requires a driving forward signal in order to generate a response. In formal terms the activity $x_{V 1}^{(2)}$ of model cells in the second processing step is formed by a non-linear combination of the FB signal $x_{V 1}^{F B}$ and the driving forward signal $x_{V 1}^{(1)}$ from the first processing step

$$
\dot{x}_{V 1}^{(2)}=-x_{V 1}^{(2)}+x_{V 1}^{(1)} \cdot\left(1+\gamma \cdot x_{V 1}^{F B}\right),
$$

where $\gamma$ is a parameter, typically in the range of 10 to 100 , to amplify the FB signal and $x_{V 1}^{(2)}$ typically ranges between zero and one. All equations and parameters appear without dimensions as we did not model the exact biophysical processes of synapses or neurons. Any paired occurrence of forward signal $x_{V 1}^{(1)}$ and FB signal $x_{V 1}^{F B}$ leads to an amplification of $x_{V 1}^{(2)}$ by an amount proportional to the product of both.

In the third processing step signals are normalized by dividing activity of a target cell by the activation summed over encoded disparities or motions. This division keeps activity in the system bounded. In combination with the modulatory enhancement this normalization realizes a biased competition that in turn deemphasizes activities for those features that did not receive any enhancement by FB signals. Formally, the activity $x_{V 1}^{(3)}$ of model cells integrates those of the second processing step $x_{V 1}^{(2)}$ summing them over all encoded disparities or motions, respectively, for each spatial location,

$$
\dot{x}_{V 1}^{(3)}=-A \cdot x_{V 1}^{(3)}+x_{V 1}^{(2)}-B \cdot x_{V 1}^{(3)} \cdot \sum x_{V 1}^{(2)} .
$$

The symbols $A$ and $B$ are parameters. Parameter $A$ is typically 0.1 , whereas $x_{V 1}^{(3)}$ ranges between zero and one. Values for parameter $B$ typically are around one. The steady-state solution of this equation is $x_{V 1}^{(3)}=x_{V 1}^{(2)} /\left(A+B \cdot \sum x_{V 1}^{(2)}\right)$ which shows the normalization property. Due to the division by the sum and assuming $B \geq 1$ the values for $x_{V 1}^{(3)}$ will not exceed an upper limit of value one.

Three-step-processing cascade for model areas V2 and MT. In the first step we use an isotropic integration from model area V1 to model area V2 or MT. During integration the spatial resolution is reduced by the factor 2.5 for V2 and 5 for MT. Note that the RF sizes in MT are about ten times larger than those of $\mathrm{V} 1{ }^{\text {iv }}$ (Angelucci et al., 2002). This reduction in resolution is achieved by convolving the input velocity space in its spatial dimensions with a Gaussian filter of appropriate size (sampling theorem) (Nyquist, 2002). Samples for every 2.5 th value for the disparity signal or every fifth value for the motion signal are taken from this Gaussian filtered signal. In cortex signal integration in MT leads to an increase in the direction tuning bandwidth (mean value $95^{\circ}$ ) compared to that of $\mathrm{V} 1$ (mean value $68^{\circ}$ ) (Albright, 1984). This increase of bandwidth is another indicator for the integrative or smoothing behavior of cells in area MT. In formal terms this integration is denoted by

$$
\dot{x}_{V 2 / M T}^{(1)}=-x_{V 2 / M T}^{(1)}+f_{\text {sample }}^{\text {rate }}\left(\left(x_{V 2 / M T}^{F F} * \Lambda_{\text {space }}\right)^{\alpha}\right) * \Lambda_{\text {disp } / \text { mot }} \text {. }
$$


For inter-area signal processing between area V1 and V2 or MT the input signal $x_{V 2 / M T}^{F F}=x_{V 1}^{(3)}$ is spatially integrated by utilizing the function $f_{\text {sample }}^{\text {rate }}$. This function implements a linear interpolation at a given the above rates. Before applying the linear interpolation, the signal is convolved by an appropriately parameterized Gaussian kernel that reduces the power of all signal frequencies above the maximum frequency divided by the sampling rate to $\sim 1 \%$ v. This integration reflects the difference between receptive field sizes in area V1 and V2 or MT (Albright and Desimone, 1987). Next, activities are smoothed over the domain of disparities or velocities by convolving with $\Lambda_{\text {disp/vel }}$. Furthermore, a nonlinear transformation of the signal is incorporated with the exponent $\alpha$, as in the Equation 11a.

In the second step of the three-stage processing cascade, FB from other model areas or an attention signal (Raudies \& Neumann, 2010) could be included in principle. However, in the current model, areas V2 or MT are the highest areas in the model and do not receive any modulating input, nor do they incorporate any attention signal. Thus,

$$
\dot{x}_{V 2 / M T}^{(2)}=-x_{V 2 / M T}^{(2)}+x_{V 2 / M T}^{(1)} \cdot\left(1+\gamma \cdot x_{V 2 / M T}^{F B}\right)
$$

simply becomes the identity $x_{V 2 / M T}^{(2)}=x_{V 2 / M T}^{(1)}$ in its steady state.

In the third step of the processing cascade, a local center-surround interaction between different disparities or velocities is computed. For this interaction similar motions or depths support each other, whereas dissimilar ones inhibit each other. More formally, the input activity $x_{V 2 / M T}^{(2)}$ is convolved with $\Lambda^{+}$, a Gaussian filter in spatial, disparity, motion direction, or log-speed domain defining the field of local support. The inhibitory field is defined by convolution of $x_{V 2 / M T}^{(2)}$ with $\Lambda^{-}$, again a Gaussian filter in the previously mentioned domains. The interaction for these filters is depicted in Figure $1 \mathrm{~b}$ for the disparity domain and in Figure 1c for the motion domain. Both, supporting and inhibiting, signals are combined in a competitive scheme by using divisive inhibition

$$
\dot{x}_{V 2 / M T}^{(3)}=-A \cdot x_{V 2 / M T}^{(3)}+x_{V 2 / M T}^{(2)} * \Lambda^{+}-B \cdot x_{V 2 / M T}^{(3)} \cdot\left(x_{V 2 / M T}^{(2)} * \Lambda^{-}\right) .
$$

The symbols $A$ and $B$ are parameters. Parameter $A$ is typically set to 0.1 , whereas $x_{V 2 / M T}^{(3)}$ ranges between zero and one. Values for parameter $B$ typically range between 1 and 10. Because of the 'soft' competition between activities representing different disparities or motions, the representation of multiple activations is possible. This center-surround interaction is the main difference to previous models of stereo (Prazdny, 1985; Pollard \& Frisby, 1990; Chen \& Qian, 2004) or motion processing (Bayerl \& Neumann, 2004; Zanker, 2005; Durant et al., 2006; Qian et al., 1994; Simoncelli and Heeger, 1998).

Table 1. Parameter values for initial signal detectors and three-step-processing cascades for signal integration in model areas V1, V2, and MT.

\begin{tabular}{llc}
\hline & \multicolumn{1}{c}{ Detection of initial disparity signals } & \\
\hline \hline Description & Parameter value & Eq. \\
\hline \hline Orientations & $\psi_{k}=k \cdot \pi / N_{\text {ori }}, k=1 \ldots N_{\text {ori }}, N_{\text {ori }}=8$ & 2 \\
\hline \multirow{2}{*}{ Gaussian filter } & $f^{\text {Gauss }}(x, y)=1 /\left(2 \cdot \pi \cdot \sigma^{2}\right) \cdot \exp \left(-\left(x^{2}+y^{2}\right) /\left(2 \cdot \sigma^{2}\right)\right)$ with & 2 \\
& $x, y \in\{-2, \ldots,+2\} \mathrm{pixel}$, and $\sigma=0.75$ pixel & \\
\hline Boundary condition & Neumann & 3 \\
\hline Normalization & $\eta_{N}=0.01$ & 4 \\
\hline Gaussian tuning & $\sigma_{c}=0.5$ & 6 \\
\hline \hline
\end{tabular}




\begin{tabular}{|c|c|c|c|}
\hline Rings of the Gabor bank & \multicolumn{2}{|c|}{$\omega_{l}=2 \cdot \pi /\left(\eta \cdot s_{1}\right) \cdot \beta^{-(l-1)}, l=1 \ldots N_{\text {ring }}, N_{\text {ring }}=6, \beta=1.5$} & 6 \\
\hline Overlap factors & \multicolumn{2}{|c|}{$o^{\Psi}=1.0$ and $o^{r}=1.25$} & 6 \\
\hline Radial standard deviation & \multicolumn{2}{|c|}{$\sigma_{l}^{\psi}=\omega_{l} \cdot \sin \left(\pi / N_{o r i}\right) \cdot o^{\psi}$} & 6 \\
\hline Tangent standard deviation & \multicolumn{2}{|c|}{$\sigma_{l}^{r}=\omega_{l} \cdot(\beta-1) /(\beta+1) \cdot o^{r}$} & 6 \\
\hline Speed to wavelength factor & \multicolumn{2}{|c|}{$\eta=2.5(\eta \geq 2$ due to the sampling theorem $)$} & 6 \\
\hline Motion directions & \multicolumn{2}{|c|}{$\phi_{m}=m \cdot 2 \cdot \pi / N_{d i r}, m=1 \ldots N_{d i r}, N_{d i r}=16$} & 8 \\
\hline Motion speeds & \multicolumn{2}{|c|}{$s_{l}=s_{1} \cdot \beta^{(l-1)}, l=1 \ldots N_{\text {ring }}, s_{1}=1.0 \mathrm{pixel} /$ frame } & 8 \\
\hline \multicolumn{4}{|c|}{ Model area V1 parameters } \\
\hline Description & V1 (stereo domain) & V1 (motion domain) & Eq. \\
\hline Nonlinearity $\alpha$ & 2 & 2 & $11 \mathrm{a}$ \\
\hline Gaussian filter $\Lambda_{\text {disp } / v e l}$ & $\begin{array}{l}\text { Horizontal disparity: } \\
\sigma_{\text {disp }}=0.75 \text { pixel and } \\
l_{\text {disp }}=5 \text { pixel }^{\mathrm{a}}\end{array}$ & $\begin{array}{l}\text { Motion speed: } \\
\sigma_{\text {speed }}=0.2 \mathrm{pixel} / \text { frame and } \\
l_{\text {speed }}=5 \mathrm{pixel} / \mathrm{frame}^{\mathrm{a}} \\
\text { Motion direction: } \\
\sigma_{d i r}=0.75 \cdot 360 / 16^{\circ} \text { and } \\
l_{d i r}=5 \cdot 360 / 16^{\circ \mathrm{a}}\end{array}$ & $11 \mathrm{a}$ \\
\hline Boundary conditions & Zero padding & $\begin{array}{l}\text { Motion speed: Neumann } \\
\text { Motion direction: Circular }\end{array}$ & $11 \mathrm{a}$ \\
\hline Feedback constant $\gamma$ & 100 & 100 & $11 \mathrm{~b}$ \\
\hline Normalization $A$ & 0.01 & 0.01 & $11 \mathrm{c}$ \\
\hline Normalization $B$ & 1 & $100 / 112$ & $11 \mathrm{c}$ \\
\hline \multicolumn{4}{|c|}{ Model area V2 and MT parameters } \\
\hline Description & V2 (stereo domain) & MT (motion domain) & Eq. \\
\hline Gaussian filter $\Lambda_{\text {space }}$ & $\begin{array}{l}\sigma_{\text {space }}=2.5 \text { pixels and } \\
l_{\text {space }}=11 \text { pixels }^{\text {a }}\end{array}$ & $\begin{array}{l}\sigma_{\text {space }}=5.0 \text { pixels and } \\
l_{\text {space }}=21 \text { pixels }^{\text {a }}\end{array}$ & $12 \mathrm{a}$ \\
\hline Sampling rate $f_{\text {sample }}^{\text {rate }}$ & 2.5 & 5.0 & $12 \mathrm{a}$ \\
\hline Nonlinearity $\alpha$ & 2 & 2 & $12 \mathrm{a}$ \\
\hline Gaussian filter $\Lambda_{\text {disp } / v e l}$ & Same as in V1 & Same as in V1 & $12 \mathrm{a}$ \\
\hline Gaussian filter $\Lambda^{+}$ & $\begin{array}{l}\text { Horizontal disparity: } \\
\sigma_{\text {disp }}=0.75 \text { pixel and } \\
l_{\text {disp }}=5 \text { pixels }{ }^{\text {a }} \\
\text { Spatial domain: } \\
\sigma_{\text {space }}=1.5 \text { pixels and } \\
l_{\text {space }}=7 \text { pixels }^{\text {a }}\end{array}$ & $\begin{array}{l}\text { Motion speed: } \\
\text { Dirac pulse, no kernel is applied. } \\
\text { Motion direction: } \\
\sigma_{d i r}=0.5 \cdot 360 / 16^{\circ} \text { and } \\
l_{d i r}=3 \cdot 360 / 16^{\circ} \text { a }\end{array}$ & $12 \mathrm{c}$ \\
\hline Gaussian filter $\Lambda^{-}$ & $\begin{array}{l}\text { Horizontal disparity: } \\
\sigma_{\text {disp }}=2 \text { pixels and } \\
l_{\text {disp }}=9 \text { pixels }^{\text {a }}\end{array}$ & $\begin{array}{l}\text { Motion speed: } \\
\sigma_{\text {speed }}=0.5 \text { pixels } / \text { frame and } \\
l_{\text {speed }}=5 \text { pixels } / \text { frame }{ }^{\mathrm{a}} \\
\text { Motion direction: } \\
\sigma_{d i r}=2 \cdot 360 / 16^{\circ} \text { and }\end{array}$ & $12 \mathrm{c}$ \\
\hline
\end{tabular}




\begin{tabular}{llll}
\hline & & $l_{d i r}=9 \cdot 360 / 16^{\circ \mathrm{a}}$ & \\
\hline Boundary conditions & $\begin{array}{l}\text { Disparity: Zero padding } \\
\text { Space: Neumann }\end{array}$ & $\begin{array}{l}\text { Motion speed: Neumann } \\
\text { Motion direction: Circular }\end{array}$ & $12 \mathrm{c}$ \\
\hline Normalization $A$ & 0.01 & 0.01 & $12 \mathrm{c}$ \\
\hline Normalization $B$ & 2 & 10 & $12 \mathrm{c}$
\end{tabular}

${ }^{a}$ The length specifies the size of the support for the filters.

\section{SIMULATION RESULTS}

The proposed model of stereo and motion processing is evaluated, first, based on physiological and psychophysical data and then applied to more realistic scenarios: images with realistic textures, scene geometries including tilts in depth, and a real video sequence of crowded motion leading to motion semitransparency. Finally, we simulate motion and stereo processing by using well-known benchmark sequences and image pairs of opaque motion and stereo setups.

Encoding a varying distance in depth or an angular difference by multiple activations and their read-out for perception. What does the neural representation for binocular or motion transparency look like, varying the distance in depth or angular difference of motion directions for independent layers? To address this question we setup quadratic $\mathrm{RDS}^{\mathrm{vi}}$ with 256 pixels side length, where correspondences are given either for a closer or a distant plane where point-correspondences for either plane are randomly selected and equally distributed amongst the planes (Figure 2a). For a large distance in depth the stimulus configuration leads to the percept of two planes, the closer one being transparent. For the model we analyzed the spatially pooled responses of area V2's disparity selective neurons. Distances smaller than or equal to $2.24 \mathrm{~min}$ arc show a single peak for the responses of the population (Figure 2b). The distance in depth is measured by angles of visual degrees in min arc, the idealized stimulus size is 256 pixels or $5 \mathrm{~cm}$ and the display distance is $60 \mathrm{~cm}$; the disparity in pixels equals $\tan ($ (angle in min arc) $/ 60 / 180 \cdot \pi / 2) \cdot 2 \cdot 60 \mathrm{~cm} \cdot 256 / 5$ pixels $/ \mathrm{cm}(2.24 \mathrm{~min}$ arc $\approx 2$ pixels). Above $2.24 \mathrm{~min}$ arc two peaks of different overlap exist, and the location of each peak corresponds to the stimulus defined depth values of the surface planes. In all cases only the horizontal disparity has been simulated.

A second experiment investigates the differences in motion direction. Here we use RDKs ${ }^{\mathrm{vii}}$ and motion direction varies between $0^{\circ}$ and $180^{\circ}$ (Figure 2e). Dots are randomly divided into two subsets with dots of each set consistently moving in a different direction. Up to $60^{\circ}$ of angular difference a single, but broad, peak exists in the response profile of motion sensitive model neurons in area MT (Figure 2f). Above $60^{\circ}$ two peaks arise in the response profile and their location coincides with the veridical motion directions, as in the case of disparity. These simulated response profiles for varying angular differences are in agreement with neurophysiology (Treue et al., 2000).

What is the perceived difference in depth or motion direction and does it differ from the peak locations in the neural representations of area V2 and MT? Humans show two effects when asked to judge the distance in these transparency stimuli. Small distances of $<2.5 \mathrm{~min}$ arc depth separation or $<25^{\circ}$ motion direction are underestimated and intermediate distances of $<12 \min$ arc or $<135^{\circ}$ are overestimated (Figure $2 \mathrm{c}$ and $2 \mathrm{~g}$ ). Assuming that the population responses match with those of motion direction selective neurons in human's area V5 (the analogue of monkey's area MT) it seems that peak locations are not directly transformed into perceived motion direction, instead the entire activation profile is involved in the read-out mechanism to interpret a particular motion stimulus (Treue et al., 2000). This difference in peak location and perceived motion or disparity motivated to model an elaborated mechanism for the read-out of the population code. Peak locations are read-out iteratively; however, previous read-outs influence succeeding ones by applying an inhibitory field (Gaussian blob) at the readout location before the next maximum location is detected. For single narrow peaked responses this inhibition leads mostly to a read-out of a single peak - the effect of attraction - depending on its width. If the single peak is broader, after the first read-out there is enough activation left for a successive read-out; 
however, the maximum location has been shifted away from the previous read-out location - the effect of repulsion. The perceived or interpreted disparities and motion directions are computed by applying this iterative read-out method with local inhibition to the individual responses which had been spatially pooled for profiles of Figure $2 \mathrm{~b}$ and $2 \mathrm{e}$. Mean and standard deviation for 50 trials are depicted in Figure $2 \mathrm{~d}$ and 2h. Simulations match well with data.

Effects of attraction and repulsion might be a general perceptual phenomenon not only limited to the domain of stereo and motion processing (Rauber \& Treue, 1999). These effects occur also in ocular stimuli constructed by line parings (Westheimer, 1986). The perceived verticality of lines as well as the offset between two line segments is influence by a superimposed line grating mask. Alignment tasks for the two line segments show effects of attraction and repulsion (Tzvetanov et al., 2007). Furthermore, attraction and repulsion occur in the stereo (Stevenson, 1991) and motion domain (Braddick et al., 2002). This suggests attraction and repulsion to be a general effect that happens to occur on the basis of population responses in various feature domains in vision.

Figure 2. Population response and perceived distance for planes at different depths and motion components with different directions. (a) The angular separation is defined as the distance in depth between two planes with a dot texture (RDS) and this distance varies in the experiment. (b) Shows the activation of disparity selective neurons for different angular separations. The activation is computed from model area V2 and pooled over the entire visual space. Dashed lines indicate the veridical depth of planes. Note that these veridical depths do not correspond with the peak locations of the activation profile in all cases. (c) As a consequence, the read out angular distance does not coincide with the veridical one; underestimation (attraction) and overestimation (repulsion) of the angular distance or depth separation occurs. (d) Comparable effects are observed for humans reporting angular distance for the same display type (Reprinted with permission???). (e) The angular difference is defined as the difference in motion direction between two randomly selected subsets of dots from a RDK. (f) Shows the population response of motion direction selective neurons from model area MT for varying angular differences. This population response is pooled over the entire visual space and motion speeds. Again, dashed lines indicate motion directions of the stimulus and these are not always coincident with the peak locations in the activation profile. (g) A read-out method interpreting the entire activation profile from (f) calculates the motion directions. Angular differences between these directions are underestimated (attraction) for small differences or overestimated (repulsion) for larger differences. Mean and standard deviation are computed for 50 trials. (h) Humans show the same effects of attraction and repulsion (Reprinted with permission ???). Note that the population responses in (b) and (d) are normalized across conditions.

What is the maximum number of depth layers or motion directions that could be processed in parallel? In the previous simulation the question about the encoding between two different layers of different depth separation or angular difference in motion directions has been studied. But, when does the representation of multiple activations for depth or motion directions deteriorate? To study this case we arranged multiple layers in depth or motion. RDSs are $768 \times 768$ pixels in size and are constructed with depth layers at horizontal disparities \pm 3 pixels (two layers); 0 and \pm 6 pixels (three layers); \pm 3 and \pm 9 pixels (four layers); $0, \pm 5$, and \pm 10 pixels (five layers) (see Figure $3 a$ ). Pixel positions were assigned to different depth layers by taking the linear index of a pixel modulus the layer number. Unlike the randomly assignment in the previous experiment this interleaved assignment of pixels allowed to create stereograms with multiple layers avoiding the formation of local clustering or cloud structures. RDKs are constructed in the same manner as in the previous simulation for varying angular difference between motion directions (see Figure $3 b$ ). In this simulation the angular difference between motions is defined as the fraction $360^{\circ} / n$ where $n$ is the number of motions. Activation profiles for both domains, stereo and motion directions look similar comparing Figure $3 \mathrm{c}$ for stereo with Figure $3 \mathrm{e}$ for motion directions. Peak signals increase as the number of encoded depth layers or motion directions decreases from two to five. Most importantly, these multiple activations are not only accomplished by integrating over the entire visual field, but also by integrating over a local neighborhood region of the visual field. This can be observed by 
looking at model V2 and MT neuron's responses. Figure 3d shows the activation of each model V2 neuron for its spatial location and horizontal disparity where all spatial locations are in a tile and tiles next to each other show different disparities. Responses for motion directions (pooled over speeds) are shown in Figure $3 \mathrm{f}$ by using the same tile organization. For two to three layers the activation in a small region of the visual space is relatively dense and is thinned out as more layers are represented (four or five in our case). Interestingly, psychophysical experiments show a 75\% detection threshold for around three to four motion directions (Felisberti \& Zanker, 2005) and the same number of different separable depth layers (Tsirlin et al., 2008). Furthermore, the perceivable number of depth layers can be improved by training (Reeves et al., 2010).

Figure 3. Representation of multiple transparently overlaid layers in depth or motions. (a) Shows a stereo image pair with two layers in depth. Note due to sampling for print the original resolution and arrangement of dots is not preserved and, thus, image fusion into stereo correspondence might not directly be possible. (b) First two frames of the motion stimulus with two different motion components. (c) Shows the population response of spatially pooled V2 signals for a RDS with the entitled number of layers in depth. Dashed lines show the horizontal disparity values of each single layer. (d) Activity maps encode the likelihood value for a disparity indicated by the axis at the bottom of the four prints. Each activity map (tile) has entries that correspond to the spatial location in the stimulus. All 25 activity maps together show the activation of all model V2 neurons of the "output layer" - after the computation of the third processing step. (e) The depicted population responses of area MT direction selectivity are pooled by summing responses over visual space and motion speeds. (f) Shows activity maps for different motion directions (columns) and an increasing number of motion components (rows) present in the stimulus. Note that the activity profiles in (c) and (e) are normalized across conditions; in contrast, intensities in (d) and (f) are individually scaled for each row.

Processing of stimuli with realistic textures, disparity, and speed gradients. So far, the processed stimuli for binocular and motion transparency did not include gradients; neither did the stimuli have realistic textures. In the next simulation these two issues are addressed. The scene consists of two planes; both tilted $45^{\circ}$ against the optical axes of the cameras (see Figure 4a). This tilt in depth leads to disparity gradients and if these planes move speed gradients occur as well. For the background plane a gravel-like texture and for the foreground plane a marble-like texture has been chosen. However, in the model their particular texture is not used to separate them. Figure $4 \mathrm{~b}$ shows the left and right camera's image. The model's simulation results are reported in Figure 4c for the disparity selective area V2 and in Figure $4 \mathrm{~d}$ and $4 \mathrm{e}$ for the motion selective area MT. Each image tile shows the spatial activation for a disparity, motion direction (pooled over speeds), or motion speed (pooled over directions). Zoomed-in versions for tiles with $6,7,8,11,12$, and 13 pixels disparity in Figure $3 \mathrm{c}$ show a transition of a maximum activity from the left to the right in visual space. This transition represents a disparity gradient caused by the tilt of planes. A similar transition occurs for motion speeds of 1.0, 1.5, 2.25, 3.38 pixels per frame in Figure $4 \mathrm{e}$ representing a speed gradient, again caused by the tilt of the planes. Motion directions show high activities for $180^{\circ}$ and $360^{\circ}$ at the location of the front plane, encoding the motion transparency. Note that the representation of disparity and speed gradients in the model does not require any specialized mechanism; instead the used population code can represent gradients in the distribution of activation across disparity or motion and spatial position. However, this does not exclude that the visual cortex has specialized representation for gradients, e.g. in the lower bank of the superior temporal sulcus for disparity gradients (Jenssen et al., 2001) or area MT (Treue \& Andersen, 1996) and higher areas for motion gradients (Sereno et al., 2002).

Figure 4. Disparity and motion speed gradients are processed for two moving tilted planes given a stereo observer. (a) Shows the configuration for the scenario: the inter-camera distance is one centimeter, the transparent front plane is six meters away (translating two centimeter per frame to the left) from the cameras $\left(40^{\circ}\right.$ vertical field of view), and the opaque back plane is 10 meters away (translating one 
centimeter per frame to the right). (b) Shows the left and right camera's image for the $9^{\text {th }}$ frame of the motion sequence. (c) Activity maps (explained in Figure 3 's caption) from model area V2. Zoomed-in versions are indicated by frames in dashed lines. High likelihood values (activities) are present for stereo correspondences, first at the farther back plane (left group of plots) and, then, at the closer front plane (right group of plots). For both groups of the three plots (left and right) there is a transition of activity from left to right that indicates the encoding of a disparity gradient that is generated by the tilted planes. (d) Shows activity maps in model area MT for motion directions whereby activity is pooled over motion speeds. Again, groups of zoomed-in versions are indicated by the two dashed frames. High likelihood values occur for leftward motion $\left(180^{\circ}\right)$ of the transparent front plane (first group) and at the same time for rightward motion $\left(360^{\circ}\right)$ of the opaque back plane (second group). (e) Pooling of area MT activity over motion directions shows likelihoods for motion speeds. Motion speeds at the location of the closer foreground plane are higher than those of the farther background plane. High likelihoods shift from left to right of the activity maps as encoded motion speeds increase. This spatial shift of high likelihoods represents a motion speed gradient that corresponds to the decrease of distance from left to right as both planes are tilted by $45^{\circ}$ with respect to the optical axes. All activity maps depict the model's state at the $9^{\text {th }}$ frame of processing the (binocular) image sequence reporting the activity after the third-processing step.

Crowded motion generates semi-transparency in motion displays. In video surveillance applications crowded motion makes it difficult to detect anomalous human behavior. We argue that an analysis on a large scale of motion crowds is related to the problem of detecting semi-transparent motion. Normal behavior of human motion in crowds includes walking in a single direction or, in terms of a crossing, in opposite direction organized in different stripes. Abnormal behavior includes crowds not moving, or single persons moving in a zigzag path through people moving in streams. Thus, normal behavioral motion leads to homogenous motion of a single component or of multiple components. If these multiple motion components are integrated over a local region of the visual field, semi-transparent motion occurs. For a model simulation we analyzed two cuts of a publicly available video from the Tokyo Shibuya crossing, see Figure 5a and 5c. Based on the motion signals in area MT we segmented each frame of the two sequences into the categories no motion, single motion, or multiple motions, depicted in Figure $5 \mathrm{~b}$ and $5 \mathrm{~d}$. In addition, we show the activity of direction selective model area MT neurons pooled over three different local regions of visual space, indicated by the circles with the numbers 1,2 , and 3 in Figure $5 \mathrm{a}$ and $5 \mathrm{c}$. For the region with no motion, in the upper half of the video cuts, all direction selective neurons weakly respond (region 1). Multiple motions (two in our case study) are represented in the lower half of the visual field where crowds form stripes in their movement (region 2). Sometimes people cluster into groups of similar motion (region 3 ). The activity in the polar histograms in Figure 5b and 5d builds up gradually over the time course of four simulated frames. The difference between the distributions of model MT neuron's activation for regions 1, 2, and 3 becomes more prominent, or in general the signalto-noise ratio is increased over the time course of four iterations. This increase in signal-to-noise ratio is caused by the spatio-temporal integration of motion signals in the model, especially its FB signaling from model area MT to V1 that leads to a disambiguation of local motion signals (Bayerl \& Neumann, 2004). A segmentation of video sequences into stationary, single, and multiple motion components is possible either by reading-out or directly analyzing the activity distributions of motion selective neurons of model area MT. This segmentation might help to detect regions of interest in video surveillance systems monitoring public places.

Figure 5. Crowded motion generates semi-transparency. Two cuts, each four frames long, of a movie from the Tokyo Shibuya crossing in Japan, provided at http://www.youtube.com/watch? $v=Z r r m 3 z z I q 3 s$ have been analyzed. (a) Shows frame 4903 of the video sequence, where persons carrying umbrellas move to the left and right and their motion is organized in stripes. This juxtaposed movement leads to patches of opponent motion overlay in local regions of the visual field. The activity distribution for motion directions (activity for speeds summed) are analyzed by pooling over the area indicated by the 
circles of 100 pixels radius in dashed line 1, 2, and 3 with the $(x, y)$ pixel coordinates $(188,129)$, (237, 347), and (349, 414), respectively. (b) Regions with zero motion (black), a single motion (gray), and multiple motions (white) are depicted for frames 4703 to 4706. Regions of motion transparency occur at the boundary between different motions and are further saptio-temporally integrated, e.g. for the region 2. In region 1 there is no motion signal and in region 3 a single motion. (c) In the same movie at frame 1821, persons move partially toward and away from the camera, again organized in lines of similar motion. Pixel coordinates for local regions 1, 2, and 3, are (89, 85), (271, 353), and (437, 342), respectively. (d) Motion semi-transparency occurs at the boundary of different motions (white regions). The distribution of activity for motion directions is shown in circular histograms, in region 1 with no motion, in region 2 with transparent motion, and in region 3 with a single motion. For both cuts motion occurs only in the lower half of images where crowds move and the camera is stationary. Note that circular histograms in $b$ and $d$ have been individually scaled and, thus, can not be directly compared.

Scenarios with opaque materials are processed with the same model. How does the model perform for stereo image pairs and image sequences with only opaque materials and non-crowded objects? For both domains, stereo and visual motion processing, a large body of test cases is provided at http://vision.middlebury.edu (Baker et al., 2011; Scharstein \& Szeliski, 2003; Scharstein \& Pal, 2007). Model simulations for these tests cases have been performed with the same parameter values as in the above simulations, with exception of the possible disparity or speed ranges which vary for these test cases. To compare simulation results in full resolution, activities of model area V1 have been read-out after the third processing step. For our stereo model 20 iterations have been applied feeding the model with the same input in each step, instead of an input from temporally successive frames. For both domains stereo and motion we use a weighted vector summation to interpret the population of disparity or motion selective neurons. This summation uses the disparity or motion as a vector and the activity as weights which are normalized by the sum of all activities for a given spatial location. The resulting disparity maps are depicted in Figure 6. All maps look like a smoothed version of the ground-truth. This is explained by the model's spatial integration and FB loop between model areas V1 and V2. So far, neither the model nor the read-out method include an explicit mechanism to preserve depth or motion defined boundaries while integrating spatial or spatio-temporal coherent stimulus components, a mechanism that is used in various grouping schemes for contour segments (Grossberg and Mingolla, 1985; Franken et al., 2006; Brox et al., 2006) and in computer vision algorithms for flow-gradient preserving integration (Horn \& Schunck, 1981; Bruhn et al., 2006). In the estimated disparity maps the left part is partly missing or blurred out. For these regions no stereo correspondences are given, as they fall into a region of the left image where the right one has no corresponding parts. Therefore, these regions are propagated from spatial locations in the left image where stereo correspondences still exist. Similarly, occluded parts, the black regions in the ground-truth disparity maps, are 'filled-in' by the model from neighboring regions with stereo-correspondences by the spatial interaction between model areas V1 and V2. Note that the disparity ranges of the model simulations have been adjusted to the interval of horizontal disparity in the stereo image pairs. The used interval of disparity values is denoted below each column of the test cases shown in Figure 6.

Figure 6. Estimation of horizontal disparity for image pairs depicting objects with opaque material properties, and no clutter of objects occurs. All image pairs are from the Middlebury benchmark for stereo computation (http://vision.middlebury.edu/stereo/data/; 2003 and 2005 data sets). Names of the stereo image pairs are written atop. A ground truth and simulated disparity map read-out from model area V1 follows. At the bottom of each test case the interval of disparities used in each simulation is given. All disparity maps are computed with respect to the left image. Horizontal disparity is encoded by the gray-value code on the right.

Simulation results for optic flow are evaluated by comparison against ground-truth in terms of angular error (AE) and endpoint error (EE), see Table 2. Errors for image sequences with low speeds are 
small, especially the EE (e.g. 'Army', 'Yosemite', or 'Mequon') because low speeds can be accurately represented in the model; in contras, larger speeds are sparsely represented, due to logarithmic sampling. Consequently, errors for image sequences with high speeds such as 'Grove', 'Urban', and 'Teddy' are larger. This is especially visible in the high EE for these three sequences (highlighted by using an italics font style). In addition the sequence 'Grove' contains many fine structures from a modeled bush moving at fast speed which are smoothed out in the model's velocity estimates; see also an earlier comment on the model's smoothness constraint. Overall, the model can process scenes with opaque materials; however, we do not claim to compete with computer vision algorithms that are optimized for this task. We emphasize that the newly proposed model architecture is capable to process transparent as well as opaque surface appearances without specific parameter changes of the model mechanisms. It is demonstrated that the architecture successfully explains experimental findings using artificial test stimuli designed to probe the perception of stereo and motion transparency. Furthermore, the model is also able to process real data and particularly suggests a new approach to treat crowding as a special case of semi-transparent motion. The final demonstration closes the range of evaluating computational stereo and motion models by demonstrating the successful processing of benchmark sequences from computer vision repositories.

Table 2. Error statistics for optic flow estimation. Motion has been computed for the $10^{\text {th }}$ frame of the Middlebury benchmark image sequences (http://vision.middlebury.edu/flow/data/) from the $7^{\text {th }}$ frame on, except for the 'Teddy' frame pair. Thus, motion is integrated over four frames. Estimated optic flow is calculated by the average vector sum based on model area V1 activity. Due to higher motion speeds in some sequences the range of speeds has been extended to eight ( $N_{\text {ring }}=7$ not counting the zero speed).

Angular error (AE) is defined as the angular difference of the estimated flow vector and ground-truth flow vector, whereas a third component of value one has been added to the $2 D$ vectors. The endpoint error (EE) is defined as the Euclidian norm of the difference vector between estimate and ground-truth. Mean value and standard deviation (SD) are computed for these errors over the entire image region, including the boundary.

\begin{tabular}{lcccccccc}
\hline Error & Army & Mequon & Schefflera & Wooden & Grove & Urban & Yosemite & Teddy $^{\mathrm{a}}$ \\
\hline \hline Mean AE (deg) & 14.5 & 11.4 & 16.8 & 11.7 & 16.6 & 27.3 & 9.77 & 37.9 \\
\hline SD AE (deg) & 12.0 & 16.9 & 21.0 & 19.4 & 24.2 & 33.4 & 7.38 & 41.0 \\
\hline Mean EE (deg) & 0.45 & 1.07 & 1.55 & 0.97 & 3.23 & 3.44 & 0.43 & 5.63 \\
\hline SD EE (deg) & 0.34 & 1.08 & 1.77 & 1.24 & 3.45 & 7.34 & 0.29 & 3.92 \\
\hline
\end{tabular}

'The 'Teddy' sequence provides only two images, thus, motion integration across three frames is not possible and estimates of the model are very poor.

\section{FUTURE RESEARCH DIRECTIONS}

Future research on binocular and motion transparency processing could combine the two pathways of stereo and motion signaling. Such an approach had been already demonstrated for opaque surface motions (Qian \& Andersen, 1997). Furthermore, physiological data shows that area MT has neurons selective for disparity (Born \& Bradley, 2005) and their role for motion transparency processing might be important (Bradley et al., 1995). Suppression effects for the anti-preferred motion direction for motion and disparity tuned neurons were strong if the stimulus depth was near the preferred depth. This suggests that opponent motion directions could be represented in parallel; however, in different depths. This parallel representation relates to studies about contextual influences on perceptual judgments about figure motion and the underlying neural responses in area MT (Duncan et al., 2000). In this study a barber-pole pattern in diamond shape has been designed that is composed of vertical stripe patterns moving horizontally. The stripe terminators are stereoscopically defined by texture panels displayed in front or behind the moving diamond. In a nutshell, the terminators, judged as intrinsic or extrinsic to the diamond stripe pattern, influence the perceptual decision of the integrated movement direction so that the extrinsic terminators discard the movement information from being integrated (see the discussion in Albright \& Stoner, 2002). The model mechanisms, especially the three step-processing cascade could be combined with the 
principles to selectively integrate neuronal responses that segregate along a particular feature dimension, such as stereo in the above described stimulus (Beck \& Neumann, 2010).

Different data show multiple activations of MT neurons for various angular differences of transparently overlaid motion directions beside the preferred motion direction (Treue et al., 2000). In the current model, MT neurons are purely selective for only motion directions and speeds. By adding a disparity selective modality to the motion sensitive neurons the possible space of interactions is extended by another dimension. Whether the proposed local center-surround interaction generalizes for such a 3D feature space defined by motion direction, motion speed, and disparity remains to be explored. Even further, it is yet an open issue whether such a model mechanism uses a localized or a distributed code. In a localized representation, each neuron would directly operate on a 3D feature representation while in the latter case motion sensitive neurons would operate on a 2D (speed, direction) velocity representation and might additionally receive and send projections from and to remote representations of disparity or stimulus depth in general (e.g., in area V2).

In the current model disparity and motion gradients can be represented without applying explicit detection mechanisms. Recent investigations demonstrate that neurons show explicit selectivity to these stimulus properties both for stereo (Jenssen et al., 2001) and motion (Sereno et al., 2002). An extension of the current model could use the gradient information contained in model areas V2 and MT representations and project them into a space of cells that are explicitly tuned to gradient information. For disparity gradients, a prototypical cell from this space could sum activity that is continuously changing in disparity and horizontal spatial location, but homogeneously integrating over vertical spatial locations, which would represent the selectivity for the tilted planes in the scenario displayed in Figure 5. Such a population of neurons explicitly representing gradients could be also useful for further read-out and separation of transparently overlaid planes, as it enables to sum activation selectively for a specific gradient plane configuration over the visual field. This summation effectively increases the signal-tonoise ratio and, thus, increases the reliability of detecting tilted planar object structures in image pairs or the image stream.

Another strategy of a gradient representation could use local operators to represent gradients locally, instead of projecting activity into a set of predefined gradient patterns. Evidence for such local mechanisms in area MT is provided by physiology (Born \& Tootell, 1992) and a modeling approach (Buracas \& Albright, 1996). In models such a mechanism is also used as motion opponent operators for ego-motion and depth estimation (Royden, 1997). However, these models do not use a biologically plausible mechanism for the detection or representation of optic flow. Thus, the gradient processing by local difference operators with detected and population encoded motions remains an open yet unresolved issue, especially considering the ambiguity provided in transparency stimuli. Experiments with a transparent rotating cylinder that is viewed frontally show that disambiguation of gradients in motion transparency processing is possible (Treue et al., 1995).

\section{CONCLUSION}

We proposed a model for binocular transparency and motion transparency processing, with a local centersurround interaction as the key mechanism. This interaction supports similar disparities or motions and suppresses dissimilar ones. This mechanism of local center-surround interaction enables to resolve noisy and ambiguous perceptual input on one hand and to allow for the stable representation of multiple activations for transparent surfaces on the other hand. This mechanism has been shown to be general enough to be applied in the disparity and motion domain; furthermore, suggesting that both modalities share a common neural mechanism.

Simulation results show that such a mechanism along with an appropriate read-out method can explain effects of attraction and repulsion for disparity and motion signals in stimuli with transparent layers in different depths or moving into different directions. Real sequences that include complex geometry and realistic surface textures are successfully processed with the same model. Besides the processing of scenes with transparent materials, scenes with fully opaque materials leading to opaque 
disparity or motion can be processed with the same model and parameters as well. This makes the model also interesting for technical applications.

\section{ACKNOWLEDGEMENTS}

The authors wish to express their gratitude to Ennio Mingolla and Isao Hayashi for comments and discussions on the manuscript. F.R. is supported in part by CELEST, a NSF Science of Learning Center (SBE-0354378 and OMA-0835976). H.N. acknowledges support by the European Community in the 7th framework program ICT-project no. 215866-SEARISE and the Transregional Collaborative Research Center SFB/TRR 62 " Companion-Technology for Cognitive Technical Systems" funded by the German Research Foundation (DFG).

\section{REFERENCES}

Adelson, E. and Bergen, J. (1985). Spatiotemporal energy models for the perception of motion. Journal of the Optical Society of America, Series A, 2, 284-299.

Albright, T. (1984). Direction and orientation selectivity of neurons in visual area MT of the macaque. Journal of Neurophysiology, 52, 1106-1130.

Albright, T. and Desimone, R. (1987). Local precision of visuotopic organization in middle temporal area (MT) of the macaque. Experimental Brain Research, 65, 582-592.

Albright, T.D. and Stoner, G.R. (2002). Contextual influences on visual processing. Annual Reviews in Neuroscience, 25, 339-379.

Angelucci, A., Levitt, J., Walton, E., Hupe, J.-M., Bullier, J., and Lund, J. (2002). Circuits for local and global signal integration in primary visual cortex. Journal of Neuroscience, 22, 8633-8646.

Baker, S., Scharstein, D., Lewis, J.P., Roth, S., Black, M.J., and Szeliski, R. (2011). A database and evaluation methodology for optical flow. International Journal of Computer Vision, 92, 1-31.

Bayerl, P. and Neumann, H. (2004). Disambiguating visual motion through contextual feedback modulation. Neural Computation 16, 2041-2066.

Beck, C. and Neumann, H. (2010). Interactions of motion and form in visual cortex - a neural model. Journal of Physiology - Paris 104, 61-70.

Black, M. and Anandan, P. (1993). A framework for the robust estimation of optical flow. In Fourth International Conf. on Computer Vision, ICCV-93, Berlin, Germany, May, 1993, pages 231-236.

Black, M. and Anandan, P. (1996). The robust estimation of multiple motions: parametric and piecewisesmooth flow fields. Computer Vision and Image Understanding, 63, 75-104.

Born, R.T. and Tootell, R.B.H. (1992). Segregation of global and local motion processing in primate middle temporal visual area. Nature, 357, 497-499.

Born, R.T. and Bradley, D.C. (2005). Structure and function of visual area MT. Annual Reviews in Neuroscience, 28, 157-89.

Braddick, O., Wishart, K., and Curran, W. (2002). Directional performance in motion transparency. Vision Research, 42, 1237-1248.

Bradley, D.C., Qian, N., and Andersen, R.A. (1995). Integration of motion and stereopsis in middle temporal cortical area of macaques. Nature, 373, 609-611.

Brox, T., Weickert, J., Burgeth, B., and Mrazek, P. (2006). Nonlinear structure tensors. Image and Vision Computing 24, 41-55.

Bruhn, A., Weickert, J., Kohlberger, T., and Schnörr, C. (2006). A multigrid platform for real-time motion computation with discontinuity-preserving variational methods. International Journal of Computer Vision 70(3), 257-277.

Bullier, J., Hupe, J., James, A., and Girard, P. (2001). The role of feedback connections in shaping the responses of visual cortical neurons. Progress in Brain Research, 134, 193-204.

Buracas, G.T. and Albright, T.D. (1996). Contribution of area MT to perception of threedimensional shape: a computational ctudy. Vision Research, 36(6), 869-887. 
Chen, Y. and Qian, N. (2004). A coarse-to-fine disparity energy model with both phase-shift and position-shift receptive field mechanisms. Neural Computation, 16, 1545-1577.

Cheng, K., Hasegawa, T., Saleem, K.S., and Tanaka, K. (1994). Comparison of neuronal selectivity for stimulus speed, length, and contrast in the prestriate visual cortical areas V4 and MT of the macaque monkey. Journal of Neurophysiology, 71(6), 2269-2280.

Cumming, B.G. and Parker, A.J. (1999). Binocular neurons in V1 of awake monkeys are selective for absolute, not relative, disparity. Journal of Neuroscience, 19(13), 5602-5618.

Danielsson, P.-E. and Segera, O. (1990). Rotation invariance in gradient and higher order derivative detectors. Computer Vision, Graphics, and Image Processing 49(2), 198-221.

Darrell, T. and Pentland, A. (1991). Robust estimation of a multi-layered motion representation. In IEEE Workshop on Visual Motion, Princeton, New Jersey, October.

Darrell, T. and Simoncelli, E. (1993). Separation of transparent motion into layers using velocity-tuned mechansims. Technical report, M.I.T. Media Laboratory, Vision and Modeling Group, Technical Report No. 244.

Daugman, J. (1985). Uncertainty relation for resolution in space, spatial frequency, and orientation optimized by two-dimensional visual cortical filters. Journal of Optical Society of America, Series A, 2, 1160-1169.

Duncan, R.O., Albright, T.D., and Stoner, G.R. (2000). Occlusion and the interpretation of visual motion: perceptual and neuronal effects of context. Journal of Neuroscience, 20, 5885-5897.

Durant, S., Donoso-Barrera, A., Tan, S., and Johnston, A. (2006). Moving from spatially segregated to transparent motion: a modeling approach. Biology Letters, 2, 101-105.

Eckhorn, R., Reitboeck, H., Arndt, M., and Dicke, P. (1990). Feature linking via synchronization among distributed assemblies: Simulations of results from cat visual cortex. Neural Computation, 2, 293307.

Edwards, M. and Nishida, S. (1999). Global-motion detection with transparent-motion signals. Vision Research, 39, 2239-2249.

Franken, E., van Almsick, M., Rongen, P., Florack, L., and ter Haar Romeny, B. (2006). An efficient method for tensor voting using steerable filters. In Leonardis, A. Bischof, H., and Pinz, A. (eds.) ECCV 2006, Part IV, LNCS 3954, pp. 228-240.

Felisberti, F. and Zanker, J. (2005). Attention modulates perception of transparent motion. Vision Research, 45, 2587-2599.

Freeman, W.T. and Adelson, E.H. (1991). The design and use of steerable filters. IEEE Transactions on Pattern Analysis and Machine Intelligence, 13(9), 891-906.

Greenwood, J. and Edwards, M. (2009). The detection of multiple global directions: capacity limits with spatially segregated and transparent-motion signals. Journal of Vision, 9, 1-15.

Grossberg, S. and Mingolla, E. (1985). Neural dynamics of perceptual grouping: Textures, boundaries, and emergent segmentations. Perception and Psychophysics 38, 141-171.

Hassenstein, B. and Reichardt, W. (1956). Systemtheoretische Analyse der Zeitreihenfolgen und Vorzeichenauswertung bei der Bewegungsperzeption des Rüsselkäfers, Chlorophanus. 2. Naturforschung Teil B, 11, 513-524.

Heeger, D. (1987). Model the extraction of image flow. Journal of Optical Society of America, Series A, 4, 1455-1471.

Horn, B. and Schunk, B. (1981). Deteriming optical flow. Artifical Intelligence, 17, 185-203.

Hubel, D.H. and Livingstone, M.S. (1987). Segregation of form, color, stereopsis in primate area 18. Journal of Neuroscience, 7(11), 3378-3415.

Hubel, D.H. and Wiesel, T.N. (1970). Stereoscopic vision in macaque monkey. Cells sensitive to binocular depth in area 18 of the macaque monkey cortex. Nature, 225, 41-42.

Janssen, P., Vogels, R., Liu, Y., and Orban, G.A. (2011). Macaque inferior temporal neurons are selective for three-dimensional boundaries and surfaces. Journal of Neuroscience, 21(23), 9419-9429. 
Johnston, A., McOwan, P., and Benton, C. (1999). Robust velocity computation from a biologically motivated model of motion perception. Proceedings of Royal Society, Series B, 266, 509-518.

Ju, S., Black, M., and Jepson, A. (1996). Skin and bones: Multi-layer, locally affine, optical flow and regularization with transparency. In IEEE Conference on Computer Vision and Pattern Recognition, CVPR'96, San Francisco, CA, June 1996, pages 307-314.

Langley, K., Fleet, D., and Atherton, T. (1992). On transparent motion computation. In Proceedings of British Machine Vision Conference (BMVC), Leeds, New York: Springer, pages 247-257.

Liu, H., Hong, T.-H., Herman, M., Chellappa, R. (Oct, 1995) Spatio-temporal filters for transparent motion segmentation. In: Proceedings of the International Conf. on Image Processing, Volume 3, pages 464-467 vol.3, 23-26, doi: 10.1109/ICIP.1995.537672.

Marr, D. and Poggio T. (1976). Cooperative computation of stereo disparity. Science, 194, 283-287.

Maunsell, J. and Essen, D. V. (1983). The connections of the middle temporal visual area (MT) and their relationship to a cortical hierarchy in the macaque monkey. Journal of Neuroscience, 3, 2563-2586.

Mechler, F., Reich, D., and Victor, J. (2002). Detection and discrimination of relative spatial phase by V1 neurons. Journal of Neuroscience, 22, 6129-6157.

Meso, A. and Zanker, J. (2009). Perceiving motion transparency in the absence of component direction differences. Vision Research, 49, 2187-2200.

Mestre, D., Masson, G., and Stone, L. (2001). Spatial scale of motion segmentation from speed cues. Vision Research, 41, 2697-2713.

Mota, C., Dorr, M., Stuke, I., and Barth, E. (2004). Categorization of transparent motion patterns using the projective plane. Journal of Computer and Information Science, 5, 129-40.

Mulligan, J. (1992). Motion transparency is restricted to two planes. Investigation Ophthalmology Visual Science (suppl.), 33, 1049.

Nover, H., Anderson, C., and DeAngelis, G. (2005). A logarithmic, scale-invariant representation of speed in macaque middle temporal area accounts for speed discrimination performance. Journal of Neuroscience, 25, 10049-10060.

Nyquist, H. (2002). Certain topics in telegraph transmission theory. In Proceedings of the IEEE, "reprinted from Transactions of the A.I.E.E, pp. 617-644, Feb. 1928", 90, 280-305.

Ohzawa, I., DeAngelis, G.C., and Freeman, R.D. (1990). Stereoscopic depth discrimination in the visual cortex: neurons ideally suited as disparity detectors. Science, 249, 1037-1041.

Orban, G.A., Kennedy, H., and Bullier, J. (1986). Velocity sensitivity and direction selectivity of neurons in areas V1 and V2 of the monkey: influence of eccentricity. Journal of Neurophysiology, 56(2), 462480.

Pollard, S.B. and Frisby, J.P. (1990). Transparency and the uniqueness constraint in human and computer stereo vision. Nature, 347, 553-556.

Prazdny, K. (1985). Detection of binocular disparities. Biological Cybernetics, 52, 93-99.

Priebe, J.N., Lisberger, S.G., and Movshon, J.A. (2006). Tuning for spatiotemporal frequency and speed in directionally selective neurons of macaque striate cortex. Journal of Neuroscience, 26(11), 29412950.

Prince, S.J.D., Cumming, B.G., and Parker, A.J. (2002). Range and mechanism of encoding of horizontal disparity in macaque V1. Journal of Neurophysiology, 87, 209-221.

Qian, N. and Sejnowski, T.J. (1989). Learning to solve random-dot stereograms of dense and transparent surfaces with recurrent backpropagation. In: Proceedings of the 1988 Connectionist Models Summer School, Morgan Kaufmann.

Qian, N. (1994). Computing stereo disparity and motion with known binocular cell properties. Neural Computation, 6, 390-404.

Qian, N., Andersen, R.A., and Adelson, E. (1994). Transparent motion perception as detection of unbalanced motion signals. iii. modeling. Journal of Neuroscience, 14, 7381-7392.

Qian, N., and Andersen, R.A. (1997). A physiological model for motion-stereo integration and a unified explanation of Pulfrich-like phenomena. Vision Research, 37(12), 1683-1698. 
Rauber, H.-J. and Treue, S. (1999). Revisiting motion repulsion: evidence for a general phenomenon? Vision Research, 39, 3187-3196.

Ramirez-Manzanares, A. Rivera, M., Kornprobst, P. and Lauze, F. (2006). Multi-valued motion fields estimation for transparent sequences with a variational approach. Rapport De Recherche Inria, number RR-5920.

Raudies, F. and Neumann, H. (2010). A model of neural mechanisms in monocular transparent motion perception. Journal of Physiology - Paris, 104, 71-83.

Reeves, A., Lynch, D., Tran, M., and Grayem, R. (2010). Multiple planes in stereo-transparency. Vision Research, 10(7), 375 (abstract).

Ringach, D. (2002). Spatial structure and symmetry of simple-cell receptive fields in macaque primary visual cortex. Journal of Neurophysiology, 88, 455-463.

Rodman, H.R., and Albright, T.D. (1987). Coding of visual stimulus velocity in area MT of the macaque. Vision Research, 27(12), 2035-2048.

Royden, C.S. (1997). Mathematical analysis of motion-opponent mechanisms used in the determination of heading and depth. Journal of Optical Society of America, A, 14(9), 2128-2143.

Scharstein, D. and Szeliski, R. (2002). A taxonomy and evaluation of dense two-frame stereo correspondence algorithms. International Journal of Computer Vision 47(1/2/3), 7-42.

Scharstein, D. and Szeliski, R. (2003). High-accuracy stereo depth maps using structured light. In IEEE Computer Society Conference on Computer Vision and Pattern Recognition (CVPR 2003), volume 1, pages 195-202.

Scharstein, D. and Pal, C. (2007). Learning conditional random fields for stereo. In IEEE Computer Society Conference on Computer Vision and Pattern Recognition (CVPR 2007).

Sereno, M.E., Trinath, T., Augath, M., and Logothetis, N.K. (2002). Three-dimensional shape representation in monkey Cortex. Neuron, 33, 635-652.

Simoncelli, E.P. and Heeger, D.J. (1998). A model of neuronal responses in visual area MT. Vision Research, 38:743-761.

Snowden, R. J., Treue, S., Erikson, R. G. \& Andersen, R. A. (1991). The response of area MT and V1 neurons to transparent motion. Journal of Neuroscience, 11, 2768-2785.

Stafstrom, C.E., Schwindt, P.C., and Crill, W.E. (1984). Receptive firing in layer V neurons from cat neocortex in vitro. Journal of Neurophysiology, 2, 264-277.

Stevenson, S.B., Comack, L.K., and Shorr, C.M. (1991). Depth attraction and repulsion in random dot stereograms. Vision Research 31, 805-813.

Thomas, O.M., Cumming, B.G., and Parker, A.J. (2002). A specialization for relative disparity in V2. Nature Neuroscience, 5(5), 472-478.

Treue, S., and Andersen, R.A. (1996). Neural responses to velocity gradients in macaque cortical area MT. Visual Neuroscience, 13, 797-804.

Treue, S., Andersen, R.A., Ando, H., Hildreth, E. (1995). Structure-from-motion: perceptual evidence for surface interpolation. Vision Research, 35(1), 139-148.

Treue, S., Hol, K., and Rauber, H.-J. (2000). Seeing multiple directions of motion - physiology and psychophysics. Nature Neuroscience, 3, 270-276.

Tsai, J.J. and Victor, J.D. (2003). Reading a population code: a multi-scale neural model for representing binocular disparity. Vision Research, 43, 445-466.

Tsirlin, I., Allison, R.S., and Wilcox, L.M. (2008). Stereoscopic transparency: Constraints on the perception of multiple surfaces. Journal of Vision, 8(5), 1-10.

Tzvetanov, T., Wirmer, A., and Folta, K. (2007). Orientation repulsion and attraction in alignment perception. Vision Research, 47, 1693-1704.

Watanabe, O. (2008). A neural model for stereo transparency with the population of the disparity energy models. Neurocomputing, 71, 3158-3167.

Watanabe, O. and Kikuchi, M. (2005). Nonlinearity of the population activity to transparent motion. Neural Networks, 18, 15-22.

Weinshall, D. (1989). Perception of multiple transparent planes in stereo vision. Nature, 341, 737-739. 
Westheimer, G. (1986). Spatial interaction in the domain of disparity signals in human stereoscopic vision. Journal of Physiology, 370, 619-629.

Zanker, J. (2005). A computational analysis of separating motion signals in transparent random dot kinematograms. Spatial Vision, 18, 431-445.

\section{ADDITIONAL READING SECTION}

Braddick, O. and Qian, N. (2001). The organization of global motion and transparency. In Zanker, M. and Zeil, J. Motion Vision - Computational, Neural, and Ecological Constraints. Springer Verlag, Berlin Heidelberg New York.

Cumming, B.G. and DeAngelis, G.C. (2001). The physiology of stereopsis. Annual Review of Neuroscience, 24, 203-38.

Howard, I.P. (2002). Seeing in Depth. Volume 1 and 2, 49 Dove Lane, Thornhill, Ontario, M3J 1P3 Canada: I Porteous.

Pack, C. C. and Born, R. T. (2008) Cortical mechanisms for the integration of visual motion. in The Senses: A Comprehensive Reference. (Oxford: Elsevier Ltd.).

Roe, A.W., Parker, A.J., Born, R.T., and DeAnglis, G.C. (2007). Disparity channels in early vision. Journal of Neuroscience, 27(44), 11820-11831.

Sperling, G. (1970). Binocular vision: A physical and neural theory. American Journal of Physiology, 83, 461-534.

\section{KEY TERMS \& DEFINITIONS}

Motion transparency: If at least two motions different in speed and or motion direction occur in the same location of the visual field, then motion transparency occurs. Naturally, this happens for surfaces of transparent material moving in front of an also moving background, e.g. the dusty windows of a bus.

Binocular transparency: If multiple surfaces at different depths occur in the same location of the visual field, then binocular transparency occurs.

Semi-transparency: In contrast to transparency, semi-transparency is generated by the spatial integration by the visual system of motions from small objects. These motions are integration in a way that they appear as two layers separated by motions. For disparity in the stereo domain the same semitransparency happens.

Random dot stereogram (RDS): Dots of a certain size, shape, and luminosity are randomly distributed on the display plane with a certain density. To generate positive disparity dots for the left image are displaced to the right according to the disparity value and with respect to their position in the right image.

Random dot kinematogram (RDK): Like in the RDS, dots are randomly distributed on the display plane. Dots are shifted between frames presented in a temporally succeeding order. Note that an RDS and RDK can be combined resulting in a stimulus containing stereo and motion attributes.

Gabor filter: This filter is defined by the combination of a cosine wave (real part) and sine wave (imaginary part) each multiplied together with a Gaussian function. For instance, a one-dimensional Gabor filter is defined by $f^{\text {Gabor }}(x)=\exp (i \cdot 2 \pi \cdot f) \cdot 1 /(\sqrt{2 \pi} \cdot \sigma) \cdot \exp \left(-x^{2} /\left(2 \cdot \sigma^{2}\right)\right)$. Transformed into the Fourier domain this filter covers a Gaussian shaped frequency window centered at the frequency $f$.

Feedback (FB): A feedback signal originates from a later stage in a chain of processing steps and enters at an earlier stage in the chain. In engineering feedback occurs for controller systems, where the controller's output signal influence is directly measured and fed back into the controller. Feedback or descending projections in cortex originate from higher areas in the hierarchy of visual processing and project to lower areas.

Primary visual area (V1): This area is the first stage of cortical 'image processing' extracting end-stops like corners, orientations of edges, spatial frequency, temporal frequency, visual motion, and disparity of stimuli. 
Second visual area (V2): This area is mainly processes form, e.g. border ownership encoding, color, and disparity; relative disparity more than absolute.

Middle temporal area (MT): A lot of evidence shows that MT is the main hub for spatially and temporally integrating visual image motion, mainly from area V1. Besides motion integration, cells in area MT show also selectivity for disparity.

${ }^{i}$ Assume the detected phase by $1 \mathrm{D}$ Gabor filters for left eye's image is denoted by $\varphi_{L}$ and the right eye's image by $\varphi_{R}$. Then the horizontal disparity is computed as $\left[\varphi_{L}-\varphi_{R}\right]_{-\pi}^{+\pi} / \omega$, where $\omega$ denotes the angular frequency of the Gabor filter and $[\cdot]_{-\pi}^{+\pi}$ a circular wrapping into the range of $\pm \pi$.

${ }^{\text {ii }}$ A simplified version of the detector could only test for shifts orthogonal to the orientation that is extracted by the Gabor filters.

iii In our implementation we solve all ODEs by using their steady-state solution. Iterations between different frames of the input sequence are iterations of these steady state solution assumed for each individual frame.

${ }^{\text {iv }}$ Note there is a difference between the increase in RF size and the magnification factor. Here, the RF size in MT is about ten times larger than that in V1, however, the magnification factor in MT is about one-fifth of that of V1 (Albright and Desimone, 1987). This difference between magnification factor and RF size may reflect the Nyquist sampling theorem, where MT applies a low-pass filter which restricts the highest spatial frequency to at least the half of the sampling frequency (Nyquist, 2002).

${ }^{v}$ Assume that the maximum normalized frequency is $\pi$, then the power in the spectrum of the Gaussian filtered signal with $\sigma$ at the sampling rate $r$ is $\sigma / \sqrt{2 \pi} \cdot \exp \left(-(\pi / r \cdot \sigma)^{2} / 2 \cdot\right)$. For instance, for the sampling rate $r=5$ we apply a Gaussian filter with $\sigma=5$ pixel the power is $\sim 1.43 \%$ of the maximum.

${ }^{v i}$ The density of black dots on white background is $25 \%$. Black dots have a size of $1 \mathrm{x} 1$ pixels.

${ }^{\text {vii }}$ Random dot kinematograms have a size of 256 × 256 pixels. White dots appear on a black background at a $10 \%$ density and are constructed by (i) randomly and uniformly distributing seed points over the entire stimulus, (ii) enlarging these points to six pixels in diameter by a the morphological dilation operation, and (iii) convolving the disc image by a Gaussian filter with three pixels support and $3 / 4$ pixels standard deviation. Dots move at a speed of five pixels per frame and the motion direction varies in the experiments. 


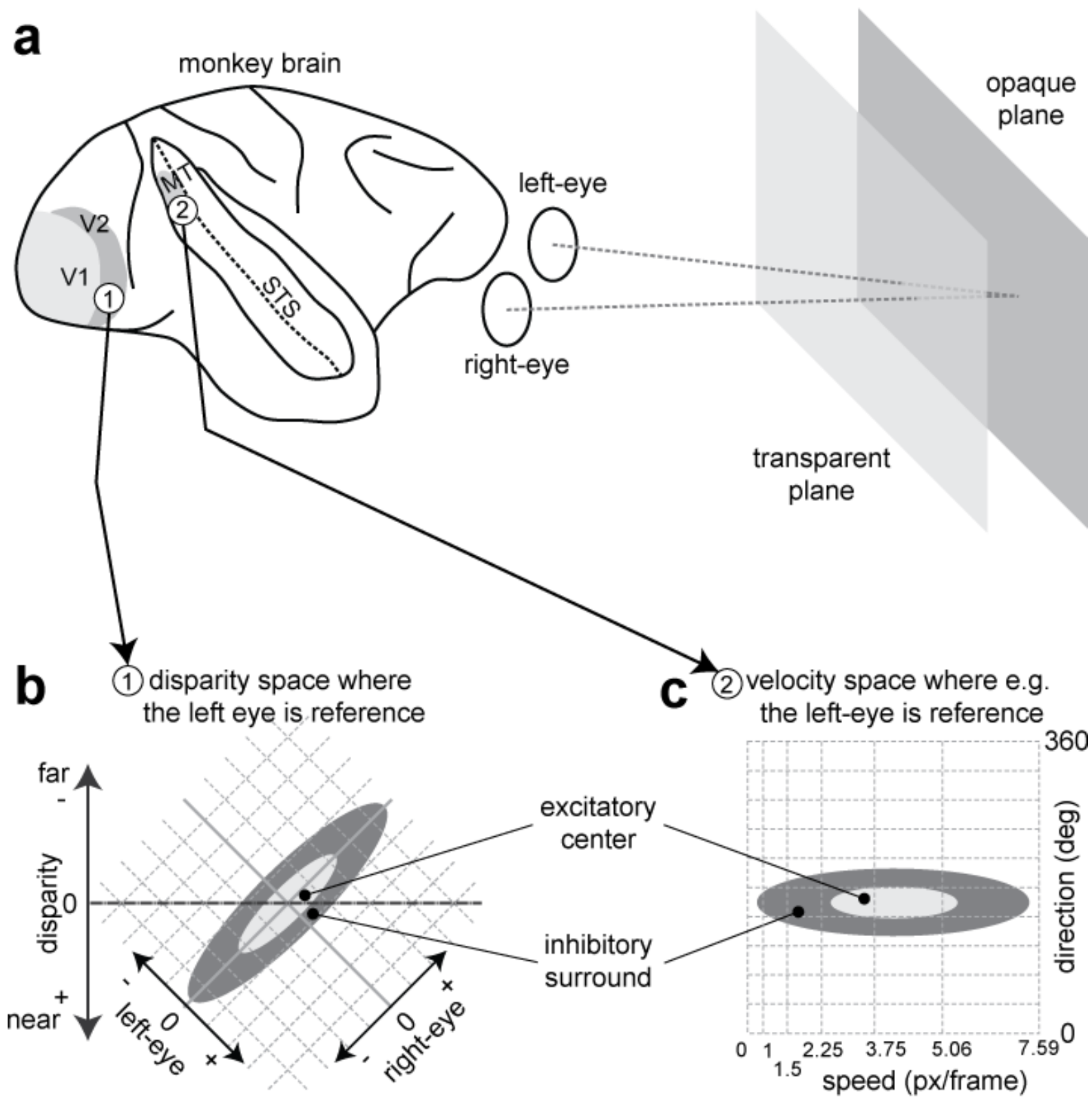

Figure 1 

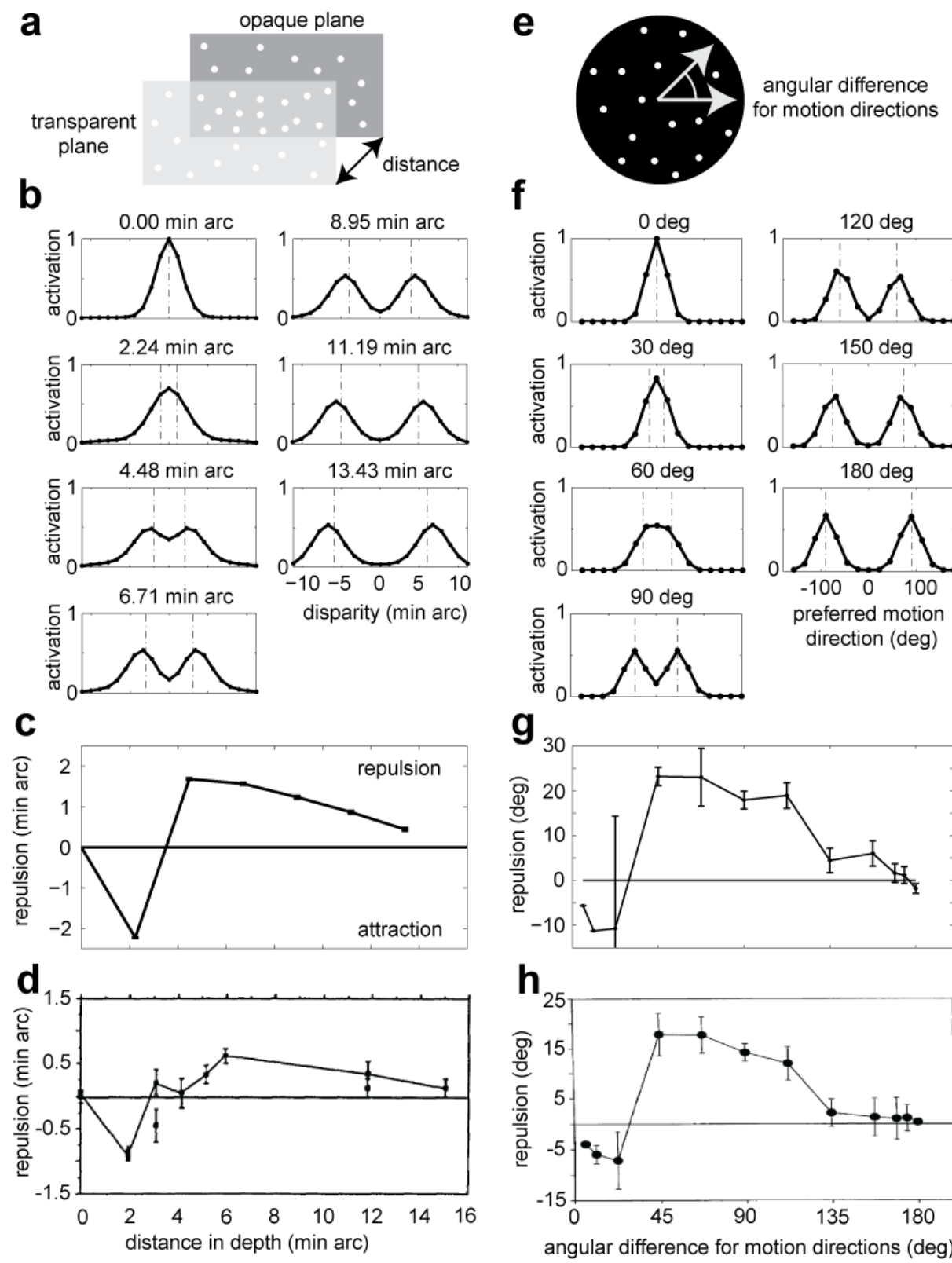

f
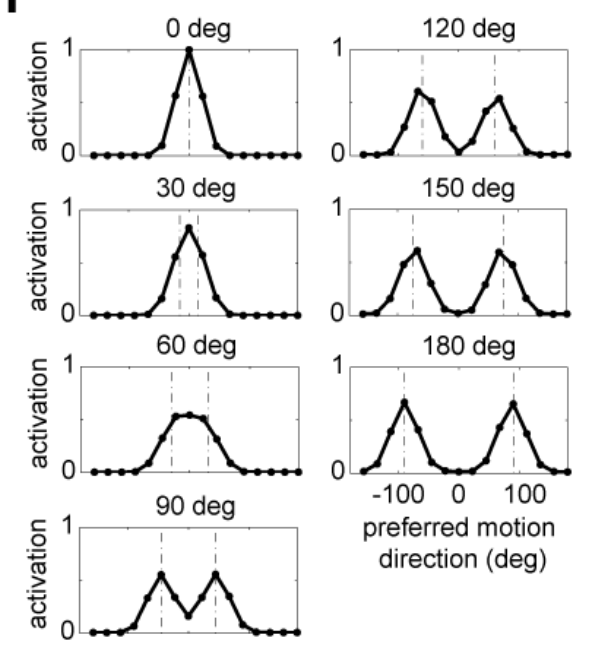

$180 \mathrm{deg}$
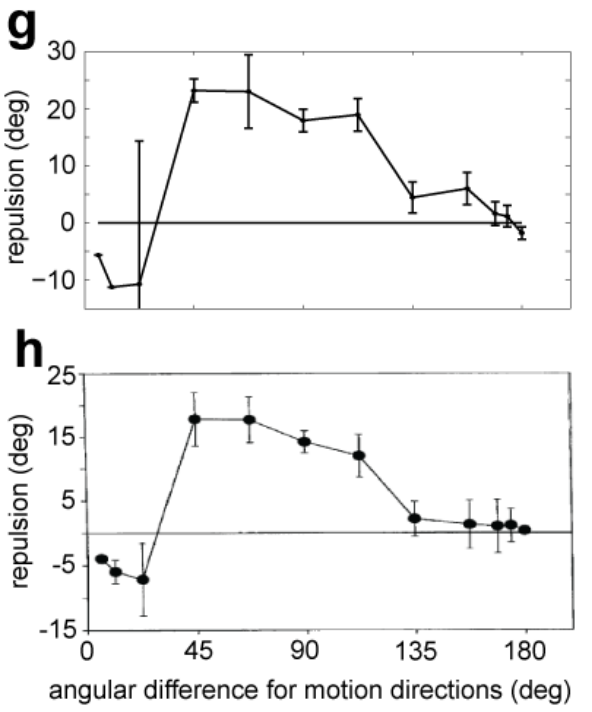

Figure 2 

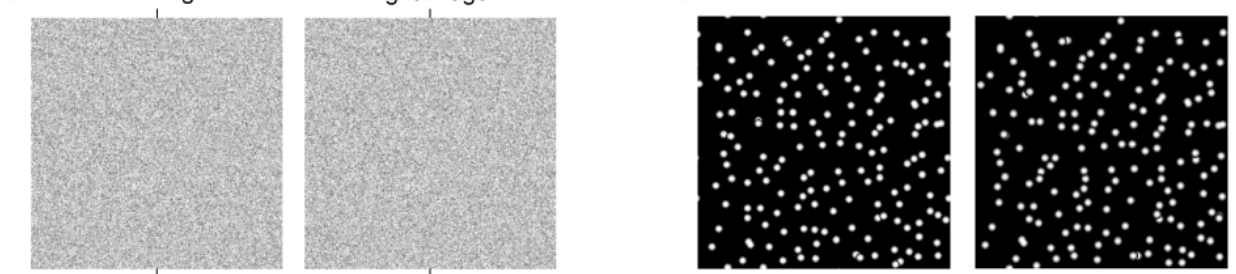

C

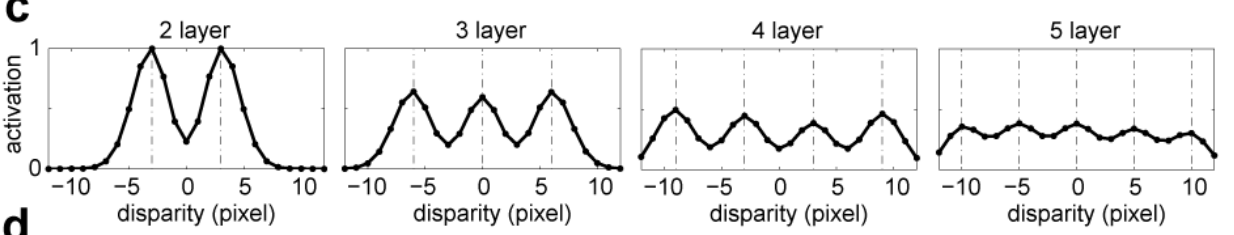

d

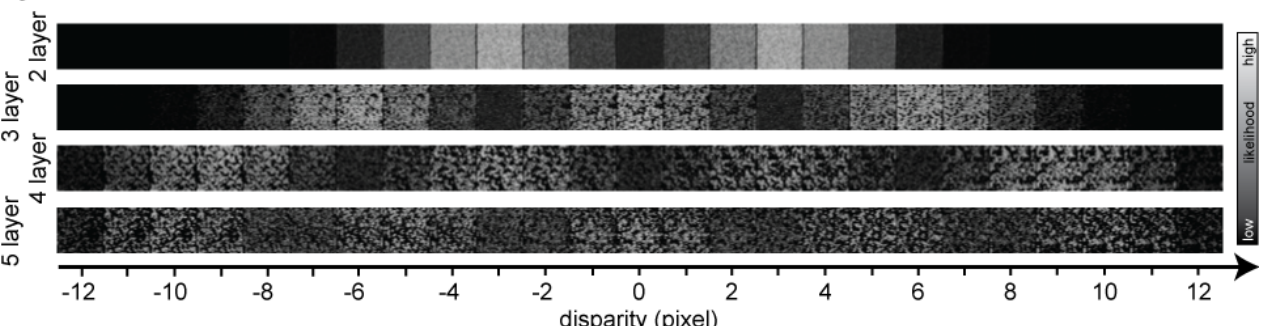

e
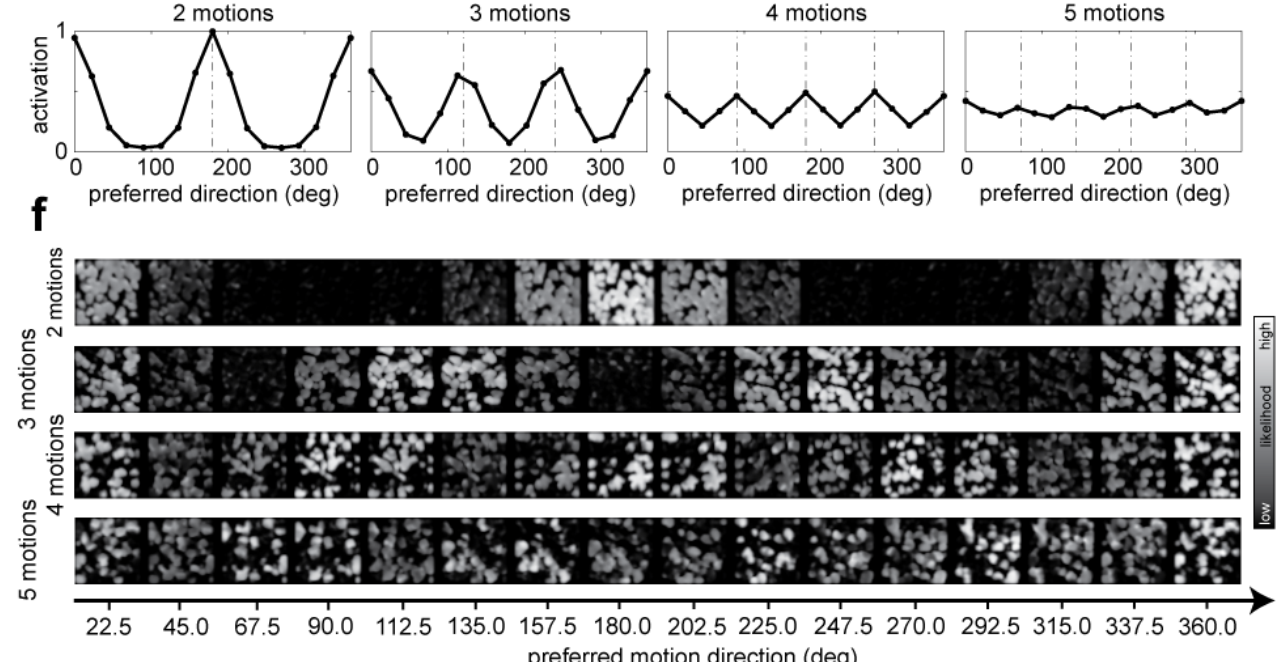

Figure 3 


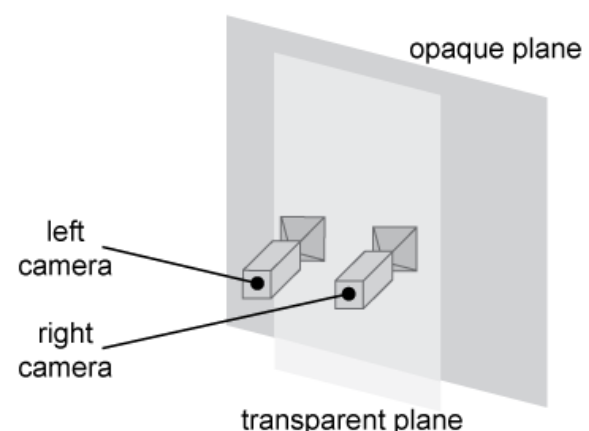

b left camera's image

right camera's image
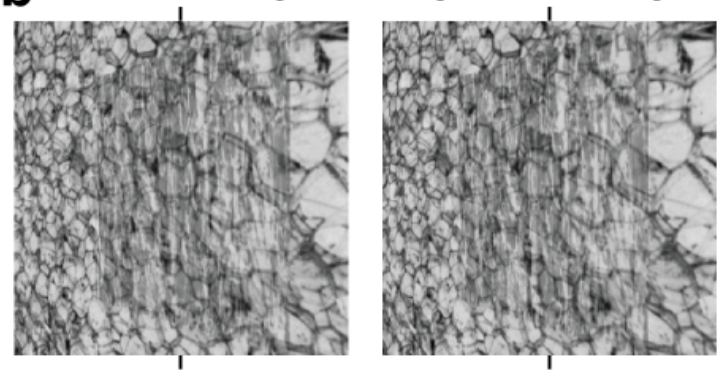

C
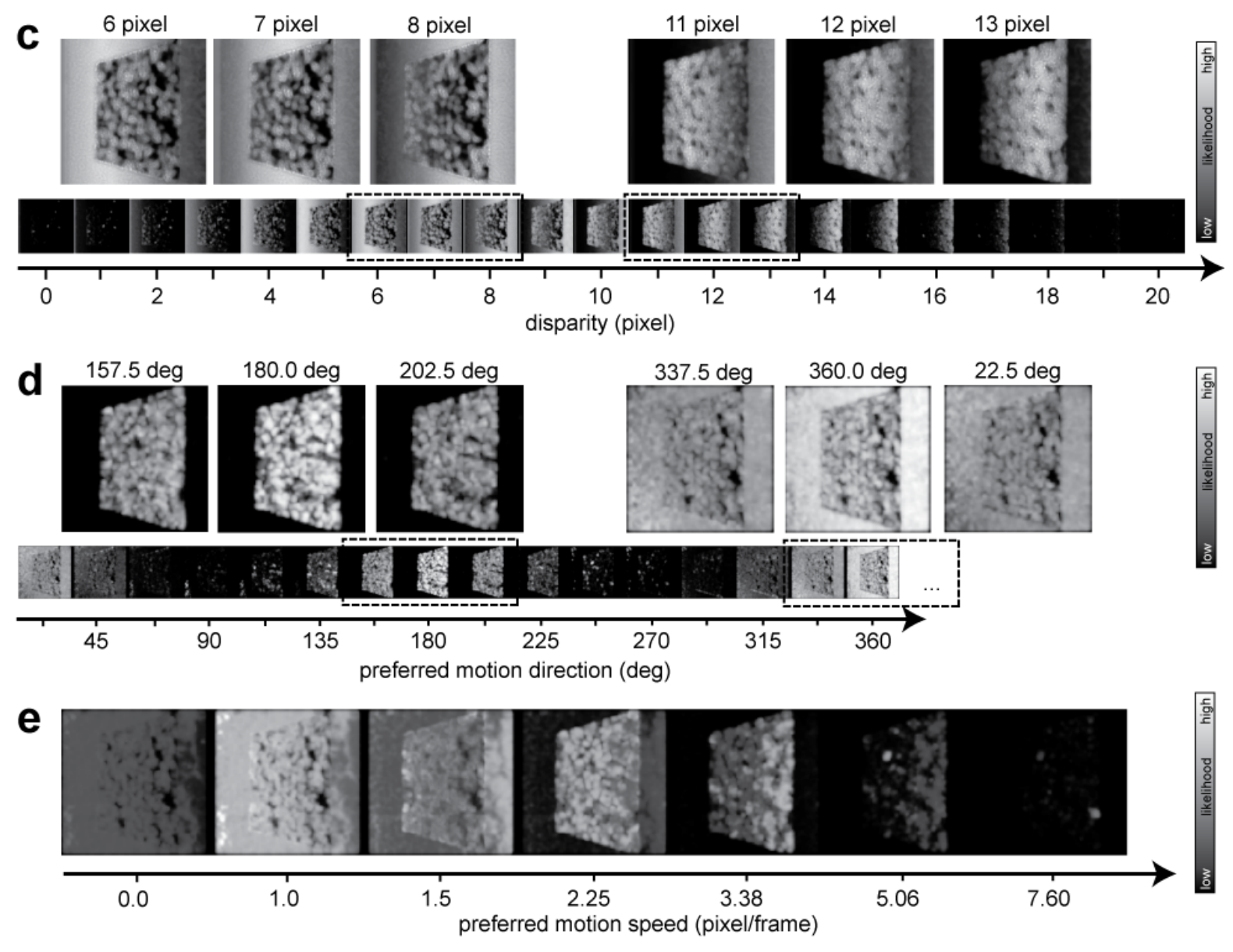

Figure 4 


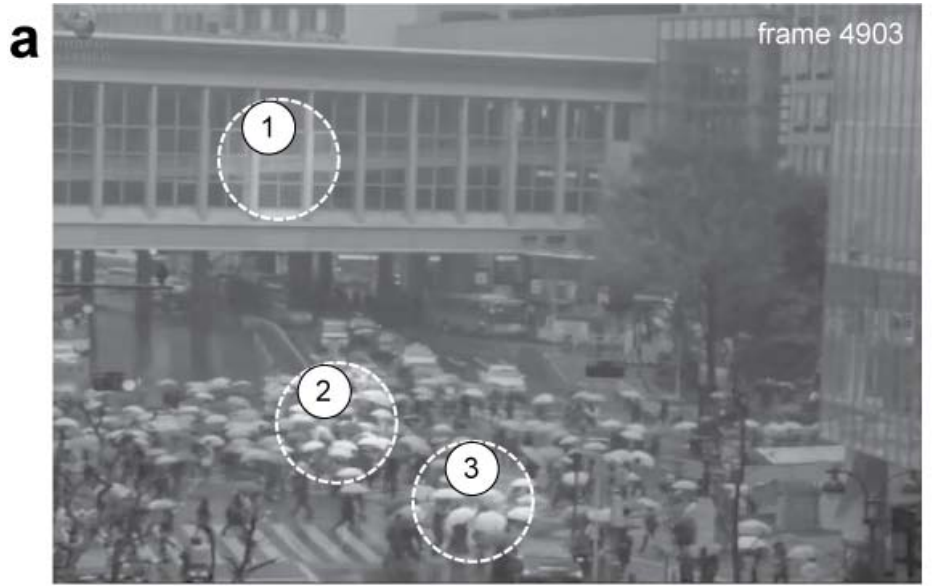

motion with zero speed

$\square$ single motion component

b
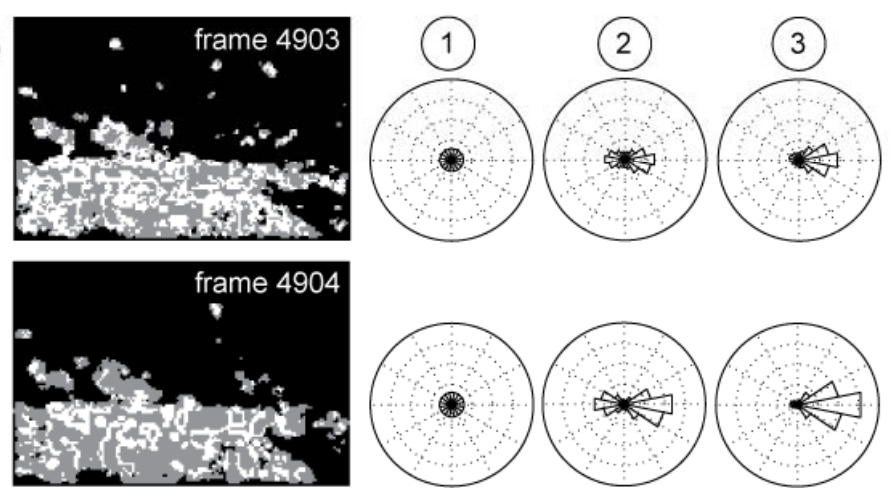

frame 4905

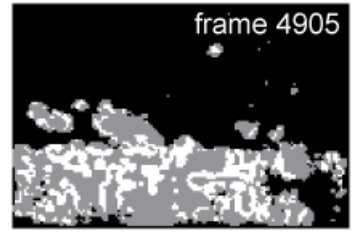

frame 4906
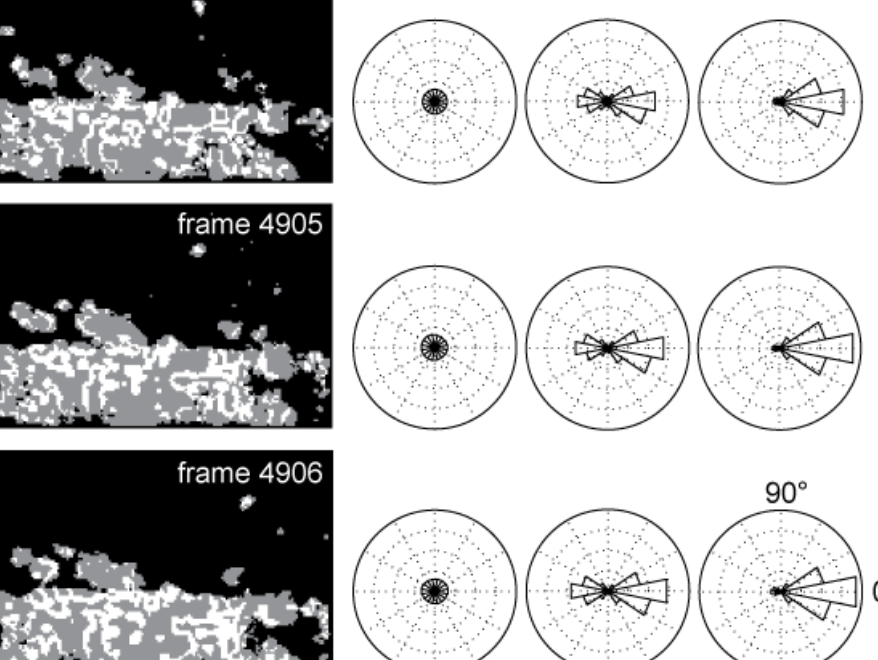

C

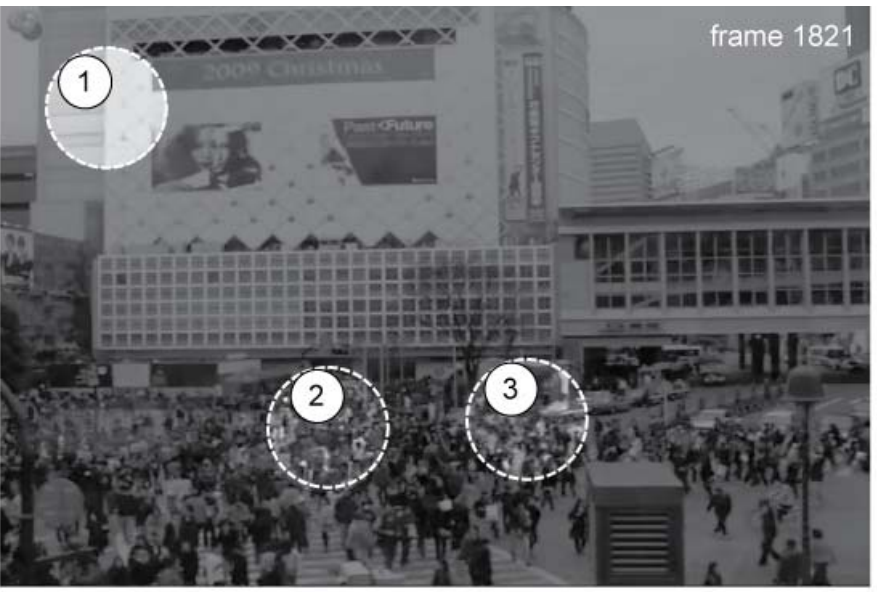

$\square$ multiple motion components
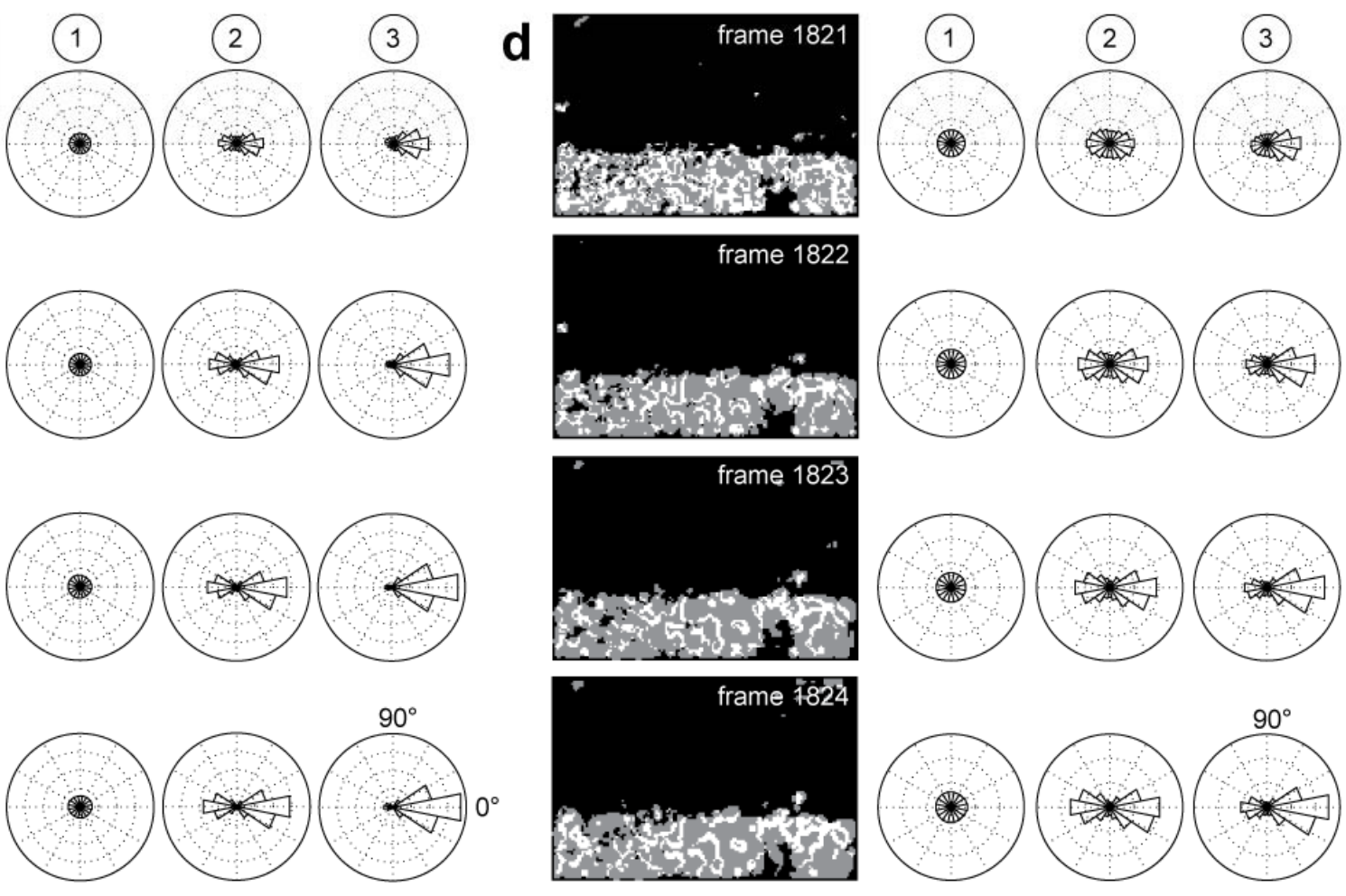

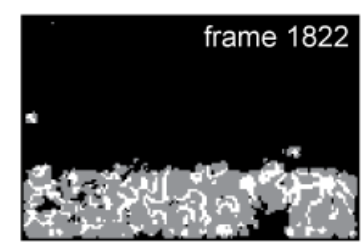

frame $182 \overline{3}$
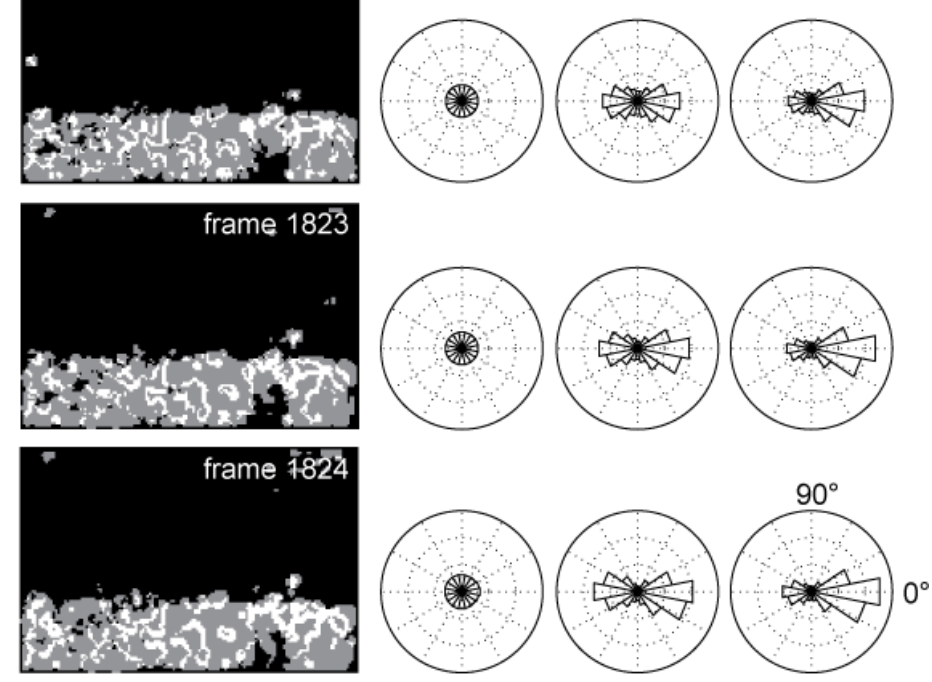

Figure 5 

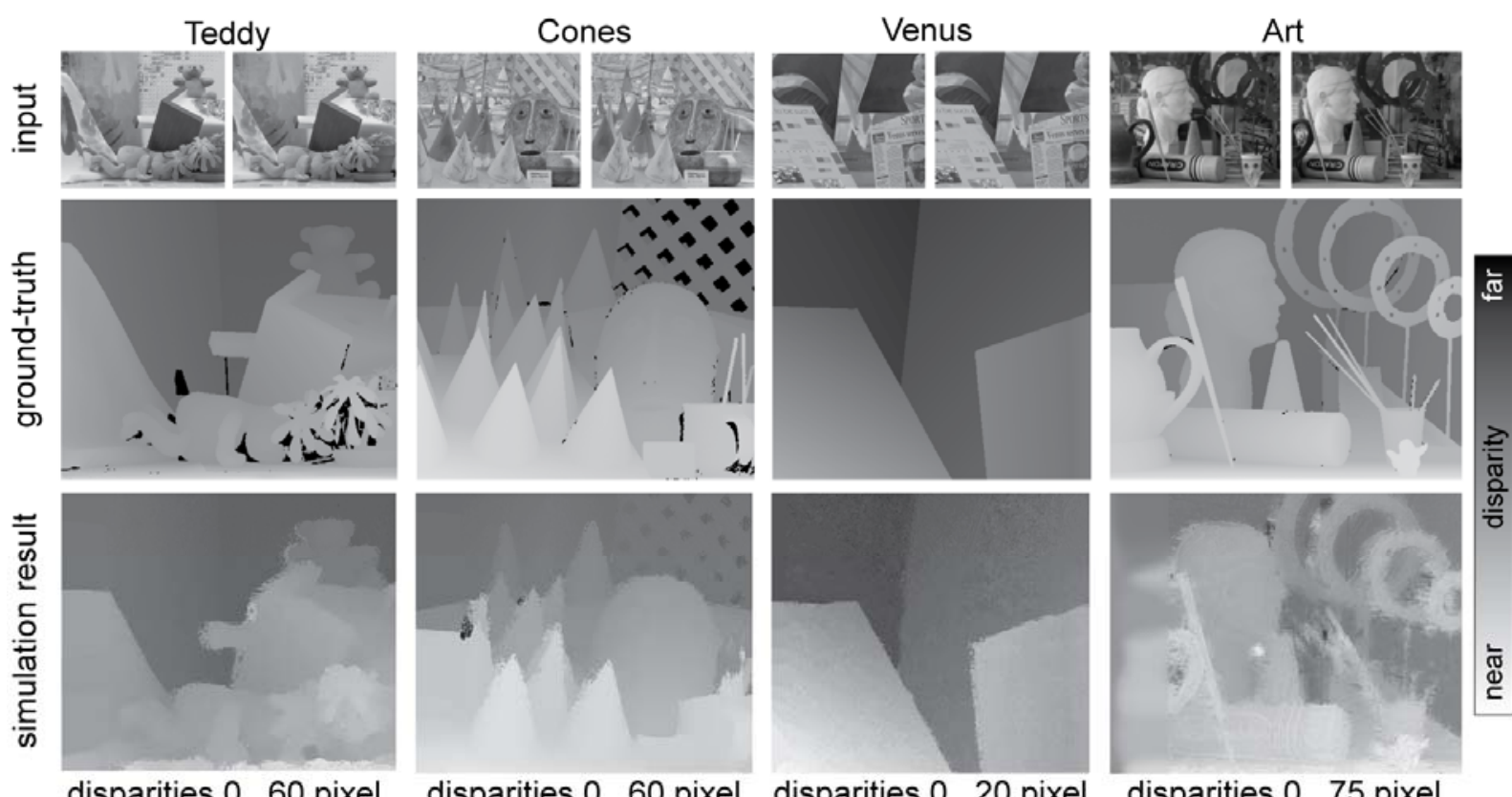

disparities $0 . .60$ pixel

disparities $0 \ldots 60$ pixel disparities $0 . \ldots 20$ pixel

disparities $0 \ldots 75$ pixel
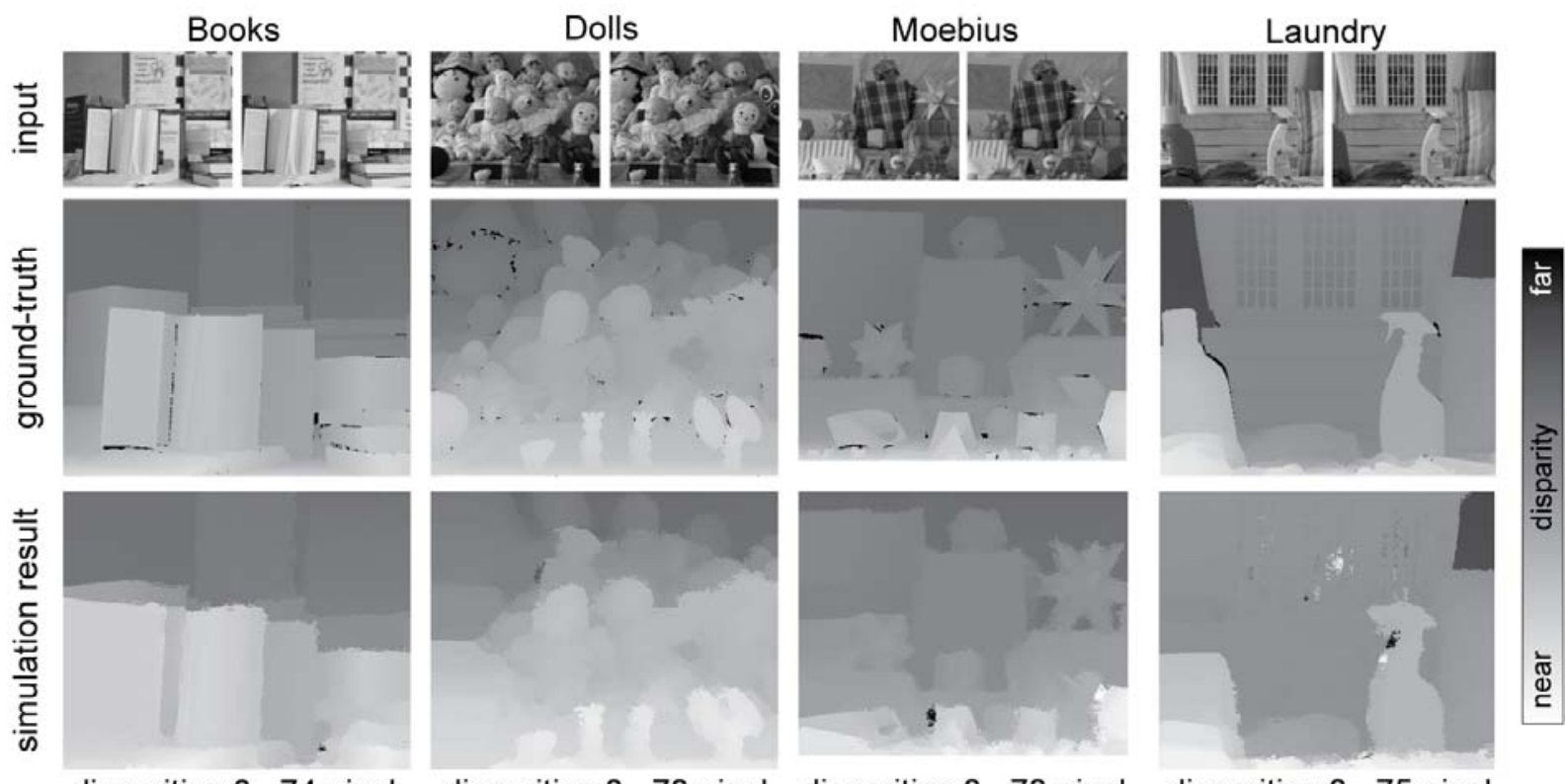

disparities $0 . . .74$ pixel

disparities $0 . .73$ pixel disparities $0 . .73$ pixel

disparities $0 . .75$ pixel

Figure 6 